\title{
Bob Jessop \\ Der Wohlfahrtsstaat im Übergang \\ vom Fordismus zum Postfordismus
}

Esist ein Gemeinplatz, daß sich der Wohlfahrtsstaat in der Krise befindet. Es stellt sich jedoch die Frage, ob es sich dabei um eine Krise im Wohlfahrtsstaat oder eine Krise des Wohlfahrtsstaates handelt. Im ersten Falle würden Teilreformen und/oder ein radikaler Umbau ausreichen, um die Funktionsfähigkeit des Wohlfahrtsstaates für die gesellschaftliche Reproduktion wiederherzustellen, ohne seine grundlegenden Organisations- und Interventionsformen zu verändern. Wenn es sich jedoch um eine Krise des Wohlfahrtsstaates handelt, bedarf es eines neuen Systems der sozialen Reproduktion. Um zwischen diesen beiden Alternativen zu entscheiden, müssen die zentralen Bestimmungen von Wohlfahrtsstaaten, die hinter ihnen agierenden politischen Kräfte, ihre Funktionen für die soziale Reproduktion sowie die Ursachen und Formen ihrer Krisen untersucht werden. Dabei sind eine ganze Reihe relevanter Faktoren zu berücksichtigen. Dieser Beitrag beschränkt sich auf drei Themenbereiche: die Formen der sozialen Reproduktion, ihr Bezug zu den langen Wellen der Kapitalakkumulation und ihre gesellschaftliche und politische Basis. Vor allem gehe ich auf die Frage ein, in welcher Weise aktuelle Veränderungen im Kapitalismus eine Beilegung der Krise im Wohlfahrtsstaat beeinflussen können. Das soll nicht heißen, daß der Wohlfahrtsstaat »in letzter Instanz« durch die Dynamik der kapitalistischen Wirtschaft bestimmt wird. Jedoch stellt diese Dynamik sicher einen der entscheidenden Faktoren für seine zukünftige Entwicklung dar.

\section{Formen und Funktionen von Wohlfahrtsstaaten}

Eine allgemeine Theorie über den Staat im allgemeinen oder über den Staat in kapitalistischen Gesellschaften kann es nicht geben (vgl. Jessop 1982; Poulantzas 1978). Es ist sogar unmöglich, von einem einzigen theoretischen Ansatz aus alle Aspekte des Staates in kapitalistischen Gesellschaften zu verstehen - selbst wenn es sich dabei, wie beim Marxismus, um ein Theoriesystem handelt, das den Anspruch erhebt, eine umfassende Analyse einer Gesellschaftsformation zu liefern. Um so weniger kann es eine allgemeine Theorie des Wohlfahrtsstaates als eines komplexen Elementes des Staates in kapitalistischen Gesellschaften geben. Bestenfalls gelangt man zu einem theoretisch informierten und historisch abgesicherten Überblick über die Formen, Funktionen und die Dynamik spezifischer Wohlfahrtsstaaten unter mehr oder weniger angebbaren Rahmenbedingungen. Und selbst dabei können noch verschiedene Ansätze, die die unterschiedlichen Aspekte des Wohlfahrtsstaates und deren Bedeutung für die soziale Reproduktion beleuchten, angewandt werden.

Meine Konzeption zur Analyse der ökonomischen und politischen Aspekte des Wohlfahrtsstaates ist »strategisch-theoretisch « angelegt. Sie geht der Frage nach, wie der Kreislauf des Kapitals und der moderne Staat gemeinsam die Formen des Kampfes zwischen den verschiedenen Klassen- und Nichtklassenkräften bestimmen und Terrains schaffen, die im Kampf zwischen diesen Kräften bestimmte Strategien gegenüber anderen begünstigen. Dieser Ansatz bezieht 
sich besonders auf die französische "Regulationsschule «", was den Kapitalkreislauf angeht, und auf die von Gramsci, Poulantzas und anderen vorgebrachten Thesen, was die Politikformen und den Staat in fortgeschrittenen kapitalistischen Gesellschaften anbelangt. Gemeinsam sind ihnen das Interesse am Zusammenspiel von Struktur und Strategie und an den Versuchen verschiedener gesellschaftlicher Kräfte, den Formen von Kapital und Staat eine Art strategischer Einheit aufzuerlegen, die sich zu spezifischen Vergesellschaftungsmustern verdichten. So hat sich die »Regulationsschule « mit den unterschiedlichen kapitalistischen Regulationsweisen und Akkumulationsregimes befaßt, die ökonomische und nicht-ökonomische Beziehungen integrieren. Gramsci und Poulantzas haben sich gleichermaßen mit den verschiedenen politischen Herrschaftsformen und der Konstruktion von »historischen Blökken « befaßt, die ein kontingentes Korrespondenzverhältnis zwischen der ökonomischen Basis und dem gesellschaftlich-politischen Überbau herstellen. Durch ihre Konzentration auf die ökonomischen bzw. auf die politischen Determinanten der Vergesellschaftung neigen beide analytischen Ansätze jedoch zur Einseitigkeit. Während sich die »Regulationsschule» primär mit dem Arbeitsprozeß und der Kapitalakkumulation befaßt, konzentriert sich der (neo-)gramscianische Ansatz auf das wechselnde Verhältnis von politischen und ideologischen Kräften. Das bedeutet, daß die theoretischen und empirischen Beziehungen zwischen den Akkumulationsregimes und den Formen politischer Hegemonie unklar bleiben, obwohl diese Beziehungen für die Frage, wie Akkumulation und/oder Hegemonie möglich sind, eine entscheidende Rolle spielen. Dieser Aufsatz geht auf diese Probleme nicht direkt ein, nähert sich ihnen aber durch eine Auseinandersetzung mit dem Wohlfahrtsstaat. Der Wohlfahrtsstaat stellt einen wichtigen Bezugspunkt für eine solche Analyse dar, da er sowohl im ökonomischen als auch im politischen System angesiedelt ist und auf ökonomische wie politische Beziehungen erheblich zurückwirkt.

\section{A. Gesellschaftliche Reproduktion und Reproduktionsstrategien}

Strategien der gesellschaftlichen Reproduktion bieten einen Ausgangspunkt für die Analyse des Wohlfahrtsstaates. Die gesellschaftliche Reproduktion kann als die Reproduktion einer bestimmten Bevölkerung ${ }^{1}$ nicht nur hinsichtlich ihrer einzelnen Mitglieder, sondern auch bezüglich ihrer Lebensfähigkeit als gesellschaftliches Ensemble definiert werden. So verstanden, ist die gesellschaftliche Reproduktion ein komplexes Phänomen mit eng verflochtenen räumlichzeitlichen, materiellen und sozialen Aspekten. Tage, Lebensläufe und Generationen markieren unterschiedliche Reproduktionszyklen von Einzelpersonen innerhalb einer bestimmten Bevölkerung; und in einem gegebenen ökonomischen System kann auch die räumliche und zeitliche Reproduktion des Gesamtarbeiters betrachtet werden. ${ }^{2}$ Die materielle Dimension der gesellschaftlichen Reproduktion bezieht sich auf diejenigen Eigenschaften und Fähigkeiten einer Bevölkerung, die sie für ihr Überleben als Gesamtarbeiter, als nationale Gemeinschaft, als militärische Macht etc. benötigt. Auch diese Eigenschaften und Fähigkeiten haben individuelle und kollektive Aspekte. Die soziale Dimension bezieht sich auf die Reproduktion der sozialen Beziehungen (Klassenbeziehungen inbegriffen), die zwischen den Individuen einer bestimmten Bevölkerung existieren. Alle drei Dimensionen hängen eng zusammen.

Die gesellschaftliche Reproduktion bringt Dilemmata und Widersprüche mit sich, so daß es keine beste Einzellösung für die Vermittlung der räumlich-zeitlichen, materiellen und sozialen Aspekte gibt. Stattdessen sollte die Reproduktion als ein Problemfeld betrachtet werden, in dem unterschiedliche Lösungen erprobt werden können. Daher kann die gesellschaftliche 
Reproduktion anhand der verschiedenen Strategien betrachtet werden, die bestimmte gesellschaftliche Kräfte in einer gegebenen Situation verfolgen, um die Reproduktionsbedingungen für sich selbst und/oder für andere Mitglieder einer Bevölkerung sicherzustellen. In kapitalistischen Gesellschaften kann man z.B. die verschiedenen Strategien untersuchen, die von Einzelpersonen und/oder Familien, Arbeitern und Gewerkschaften, Unternehmen und Unternehmerverbänden, freiwilligen Vereinigungen und pressure groups, staatlichen Stellen auf zentraler und lokaler Ebene, mit bestimmten Bereichen der Reproduktion befaßten Berufsgruppen, internationalen Organisationen usw. verfolgt werden. Der Nationalstaat ist ganz offensichtlich ein fundamentaler Ort für die gesellschaftliche Reproduktion, aber seine Formen und Funktionen müssen mit anderen Orten und Strategien sowohl inner- wie außerhalb seiner Grenzen in Beziehung gesetzt werden.

Die Formen der gesellschaftlichen Reproduktion unterscheiden sich von Gesellschaft zu Gesellschaft und werden immer von nichtökonomischen Faktoren überdeterminiert. Selbst in kapitalistischen Gesellschaften werden sie durch so unterschiedliche Formen wie den Polizeistaat, den Rechtsstaat und den Woblfabrtsstaat vermittelt, die jeweils ganz unterschiedlich geartete Verbindungen zu den Marktkräften und zu den Institutionen der bürgerlichen Gesellschaft ausprägen. Darïber hinaus kann selbst innerhalb der einen oder anderen Form die Priorität z.B. auf die ökonomische, militärische oder politische Reproduktion gelegt werden. Davon ausgehend, schafft der Kapitalkreislauf sehr wohl erhebliche Probleme bei der gesellschaftlichen Reproduktion, und diese liefern einen wichtigen Bezugspunkt für die Analyse von Arenen und Strategien der Reproduktion.

\section{Die gesellschaftliche Reproduktion und das Lobnverbältnis}

Aus der Perspektive der gesellschaftlichen Reproduktion liegt das entscheidende Merkmal des Kapitalverhältnisses in der Kommodifizierung der Arbeitskraft. Denn dies heißt, daß die gesellschaftliche Reproduktion in erster Linie durch das Lohnverhältnis vermittelt bzw. darauf ausgerichtet ist. Die gesellschaftliche Reproduktion ist insofern durch das Lohnverhältnis vermittelt, als die Arbeiter ihre Arbeitskraft gegen Lohn tauschen und diesen dazu benutzen, die Mittel zur Reproduktion ihrer Arbeitskraft zu kaufen. Dies ist zugleich der Ausgangspunkt für Wohlfahrtspolitik und für Sozialgesetze. So gehen Wohlfahrtssysteme in kapitalistischen Gesellschaften gewöhnlich davon aus, daß arbeitsfähige Erwachsene selber für ihren Unterhalt sorgen können und müssen (üblicherweise durch abhängige oder unabhängige Beschäftigung) bzw. zusammen mit Kindern oder anderen abhängigen Personen von anderen Mitgliedern ihrer Familie oder ihres Haushalts unterstützt werden (s. Zacher, 1985).

Das Lohnverhältnis ist eine von mehreren verwandten Formen des Kapitalverhältnisses: andere sind die Preis-, Geld-, Kapital-, Kredit- und Steuerformen. Das Kapitalverhältnis hängt darüber hinaus mit einer Reihe spezifischer gesetzlicher und politischer Formen zusammen (s. Tuschling 1975; Zacher 1985). Alle zusammen bilden die Matrix, in die und mittels derer der Staat in kapitalistischen Gesellschaften eingreift, um die gesellschaft liche Reproduktion sicherzustellen. Es müssen also nicht nur die ökonomischen Aspekte der staatlichen Intervention, sondern auch die gesetzlichen und administrativen Formen ihrer Durchführung in Betracht gezogen werden. Im Gegenzug offenbaren sich innerhalb und durch diese Matrix viele der entscheidenden ökonomischen und politischen Aspekte der Krise des Wohlfahrtsstaates. Als Reproduktionsmechanismus schafft die Lohnform den einzelnen Arbeitern und Haushalten zahlreiche Probleme, und sie enthält darüber hinaus Dilemmata und Widersprü- 
che für die gesellschaftliche Reproduktion im allgemeinen. Diese Probleme und Widersprüche liefern die Brennpunkte für unterschiedliche Strategien der individuellen und gesellschaftlichen Reproduktion und schaffen Raum für sozialpolitische staatliche Intervention. ${ }^{3}$ Wir betrachten diese Probleme zunächst vom Standpunkt der einzelnen Lohnarbeiter und der von dem Lohneinkommen Abhängigen aus und danach allgemeiner vom Standpunkt der Reproduktion des Gesamtarbeiters und der gesellschaftlichen Verhältnisse.

Zunächst kann der Lohn zur Deckung der Kosten der für die tägliche, lebenslange oder intergenerationelle Reproduktion notwendigen Waren und Dienstleistungen unzureichend sein. Am problematischen Verhältnis zwischen »individuellem Lohn« und »Familienlohn«(Krätke 1983) wird dies besonders deutlich. Jedoch können sich auch Unterbrechungen in der Beschäftigung etwa durch Rationalisierung, Krankheit, Unfallfolgen oder Schwangerschaft auf den einzelnen Arbeiter ungünstig auswirken. Eine der Strategien gegen diese "primäre Armut " ist die Entwicklung von Formen der Einkommenssicherung, die über kürzere oder längere Zeit den kapitalistischen Lohn ersetzen oder ergänzen. Andere staatliche Strategien umfassen die gesetzliche Festlegung von Mindestlöhnen, die staatliche Förderung von Gewerkschaften und öffentliche Arbeitsbeschaffungsprogramme. Auch individuelle oder familienorientierte Strategien (z.B. Rückhalt durch die erweiterte Familie) und/oder gemeindliche, kooperative und philanthropische Lösungen sollten nicht außer Acht gelassen werden.

Zum zweiten ist die Arbeitskraft als Ware eine Fiktion. Und zwar in dreierlei Hinsicht: sie wird nicht innerhalb einfacher Warenproduktion oder eines kapitalistischen Arbeitsprozesses hergestellt, ihre Produktion unterliegt nicht direkt dem Wertgesetz, und schließlich muß ihre Reproduktion, da es sich um lebendige Arbeit handelt, durch die aktive Mitarbeit der Arbeitskraft selbst vermittelt werden. Inter alia heißt das, daß es vorkommen kann, daß Lohnarbeiter ihre Löhne nicht dafür ausgeben, um ihre Arbeitskraft gemäß den Anforderungen des Kapitals zu reproduzieren. ${ }^{4}$ Eine Lösung für das zweite Problem ist die Sozialarbeit, um auf die Familie einzuwirken und/oder eine Gesetzgebung zur Qualitätskontrolle von Waren und Dienstleistungen sowie Zwang zur und/oder Anreiz für die Einhaltung der Reproduktionsnotwendigkeiten.

Drittens können nicht alle für die Reproduktion der Arbeitskraft notwendigen Bedingungen zwangsläufig am besten - wenn überhaupt — durch Marktmechanismen bereitgestellt werden. Dieses Problem kann von den beiden Seiten des Kapitalverhältnisses aus betrachtet werden: vom Kapital im allgemeinen und vom Gesamtarbeiter aus. So kann es für Einzelkapitale unprofitabel sein, alle für die erweiterte Reproduktion des Kapitals im allgemeinen benötigten Gebrauchswerte als Waren herzustellen - die für die Reproduktion der Arbeitskraft benötigten Gebrauchswerte inbegriffen. Umgekehrt kann das Ergebnis der Reproduktionsentscheidungen eines einzelnen Arbeiters im Widerspruch zur Reproduktion des Gesamtarbeiters stehen. Darüber hinaus hängt der Preis für wesentliche Gebrauchsgüter wie z.B. die Wohnung häufig von Faktoren ab, die mit ihrem Produktionspreis nur wenig zu tun haben (s. Krätke 1983). Das kann für den Arbeiter den Zugang über den Markt unvorhersehbar und/oder teuer machen. Eine Lösung für diesen dritten Problemkreis könnte in staatlicher Regulierung und/oder in einer marktunabhängigen Zuweisung der für die gesellschaftliche Reproduktion nötigen Mittel bestehen.

Da viertens die gesellschaftliche Reproduktion qua Lohnform davon ausgeht, daß sich die Lohnempfänger um von ihnen abhängige Personen kümmern, entstehen immer dann Probleme, wenn die Beziehung zwischen dem Lohnempfänger und den von ihm Abhängigen in die Brüche geht. Das kann entweder durch die Auflösung des Haushalts wegen Todes, Schei- 
dung oder Trennung geschehen oder dadurch, daß es den lohnabhängigen Haushalten nicht gelingt, sich fest einzurichten oder im Ruhestand für ihr Auskommen zu sorgen.

Mehr auf den Gesichtspunkt der Reproduktion des Gesamtarbeiters als auf die Problematik einzelner Haushalte bezogen, muß für die Verwirklichung der Kapitalinteressen im allgemeinen auf dem Arbeitsmarkt ein bestimmtes Kräfteverhältnis herrschen. Dieses Kräfteverhältnis bringt eine Reihe Dilemmata mit sich, die von der Tatsache herrühren, daß der Lohn sowohl zu den Produktionskosten zählt als auch eine Revenue - und somit Nachfragequelle ist (vgl. Müller und Neusüss 1970) und daß eine »Reservearmee « von Arbeitern für das Kapital sowohl vorteilhaft wie auch nachteilig ist (vgl. Offe 1985). Der Umgang mit diesen Dilemmata muß außerhalb des Kapitalkreislaufs angesiedelt werden und wird üblicherweise zum Brennpunkt staatlicher Strategien im Bereich der Arbeits- und Sozialpolitik.

\section{B. Wohlfabrtsstaat und gesellschaftliche Reproduktion}

Bislang haben wir uns auf die Spannungen, Dilemmata und Widersprüche konzentriert, die das Lohnverhältnis als dominanter Mechanismus zur Reproduktion der Arbeitskraft mit sich bringt. Die Eigenschaften und Grenzen des Wohlfahrtsstaates müssen jedoch auch mit dem politischen System in kapitalistischen Gesellschaften in Beziehung gesetzt werden. Die wichtigsten Charakteristika dieses politischen Systems sind das strukturelle und strategische Primat eines globalen Systems von Nationalstaaten sowie die zentrale Bedeutung von »nationalen«Interessen als innenpolitischer Bezugspunkt. Genauer gesagt, besteht die Eigenart des kapitalistischen Nationalstaates in seiner institutionell festgelegten Trennung vom Kern der kapitalistischen Produktion, in seiner Form als Steuerstaat, in der Rolle des Geldes als ökonomischer Matrix für seine Handlungen und in der Rolle, die das Recht und die rational-legale Bürokratie als administrative Matrix für diese Handlungen spielen. Welche Formen die politische Repräsentation, die bestimmte Herrschaftsformen charakterisieren, auch im einzelnen annehmen mag, müssen sich doch die Kräfte, die auf einen dauerhaften politischen Einfluß im staatlichen Bereich abzielen, um die politische, intellektuelle und moralische Führung sowie um die Kontrolle über das Gewaltmonopol bemühen.

Die Form des Wohlfahrtsstaates muß mit diesen typischen Merkmalen des kapitalistischen politischen Systems als Ganzem in Bezug gebracht werden. Wohlfahrtsstaaten funktionieren vor allem im Kontext des Systems von Nationalstaaten und sind der Hauptaustragungsort von Kämpfen um die innerstaatliche Hegemonie. Im allgemeinen richtet sich Sozialpolitik nicht direkt an der Organisation der kapitalistischen Produktion aus, sondern befaßt sich mit deren gesellschaftlichen Vorbedingungen und Folgen. Auch die sozialpolitischen Aktivitäten werden gewöhnlich durch Gesetzgebung und Geld vermittelt. In den Anfängen der Wohlfahrtspolitik im ausgehenden 19. Jahrhundert wird dies besonders deutlich: sie befaßte sich mit der Sozialversicherung für Arbeitsunfälle, Krankheit, Alter und später mit Arbeitslosigkeit und bediente sich üblicherweise der formalen, rational-legalen, bürokratischen Verwaltung von Geldzahlungen. Die spätere Entwicklung des Wohlfahrtsstaates eröffnete oft in wachsendem Maße Ermessensspielräume in der Sozialgesetzgebung sowie in der Versorgung mit Dienstleistungen, die nur schwer nach den formalen Prinzipien der Finanzbuchhaltung zu kontrollieren waren. Die Probleme, die sich daraus für den Wohlfahrtsstaat ergeben, werden weiter unten genauer beleuchtet. Jedoch soll jetzt schon darauf hingewiesen werden, daß die überwiegende Mehrzahl der Sozialausgaben noch immer durch Geldzuweisungen erfolgt, die den formalen und rechtlichen Regeln der Finanzbuchhaltung unterliegen. 


\section{Zentrale Bestimmungen des Woblfabrtsstaates}

"Ein 'Wohlfahrtsstaat' ist ein Staat, in dem die organisierte Macht vorsätzlich dazu benutzt wird, um (durch Politik und Verwaltung) das Spiel der Marktkräfte in mindestens drei Richtungen zu modifizieren: erstens, indem Einzelpersonen und Familien ein vom Marktwert ihres Vermögens unabhängiges Minimaleinkommen zugesichert wird; zweitens durch Verringerung der sozialen Unsicherheit, indem Einzelpersonen und Familien in die Lage versetzt werden, bestimmten sozialen Wechselfällen wie Krankheit, Alter und Arbeitslosigkeit zu begegnen, die ansonsten zu Krisen von Einzelnen und Familien führen; und drittens, indem allen Bürgern unabhängig von Stand oder Klasse die best möglichen Standards bei einer vereinbarten Anzahl sozialer Dienstleistungen garantiert werden.«(Briggs 1961, S. 228)

Diese Definition wirft ein Schlaglicht auf drei zentrale Problemfelder des Wohlfahrtsstaates, vergißt jedoch einen vierten, ebenfalls entscheidenden Aspekt seiner Organisation. Erstens bringt die Beziehung zwischen Marktkräften und Sozialpolitik Spannungen, Dilemmata und Widersprüche mit sich. Das Verhältnis zwischen Markt und Staat muß mithin als problematisch, konfliktreich und instabil angesehen werden. Zweitens: während die Marktkräfte die eine Achse des Wohlfahrtsstaates und seiner Entwicklung darstellen, bildet die Familie als Herzstück der sozialen Reproduktion eine zweite Achse. Bei der Erklärung von Veränderungen in der Form der gesellschaftlichen Reproduktion müssen Veränderungen in Struktur und Stabilität der Familien miteinbezogen werden. Obwohl sich, drittens, viele moderne Wohlfahrtsstaaten zu den »bestmöglichen Standards« verpflichten, enthält deren Fixierung doch immer eine »historische und moralische Dimension«. Überdies hat es diese Verpflichtung nicht immer gegeben, und sie ist auch nicht in allen modernen Staaten anzutreffen. Sie hängt stark mit der Entwicklung der Wohlfahrtsstaaten während der fordistischen Epoche zusammen; ihr Überleben in einem post-fordistischen Zeitalter ist jedoch fraglich. Und schließlich vernachlässigt diese Definition durch ihre Konzentration auf die vom Wohlfahrtsstaat bereitgestellten Sozialleistungen die verschiedenen Formen, in denen diese Leistungen angeboten werden. Diese Formen hängen nicht nur stark von den politischen $\mathrm{Zu}$ sammenhängen $a b$, in denen sich der Wohlfahrtsstaat entwickelt hat, sondern sie haben auch einen dauerhaften Einfluß auf seine Funktionen für die gesellschaftliche Reproduktion sowie auf seine Rolle bei der Veränderung des politischen Kräfteverhältnisses.

\section{Soziale Sicherheit und Woblfabrtsstaat}

Die Wohlfahrtspflege kann unterschiedliche Formen annehmen, und es sind verschiedene Typologien entwickelt worden, um diese zu charakterisieren. ${ }^{6}$ Die von Esping-Andersen (1986) entwickelte ist für unsere Zwecke besonders geeignet, da sie nicht nur die strukturellen Merkmale des Wohlfahrtsstaates berücksichtigt, sondern auch ihre Genese in den Klassenkräfteverhältnissen, sowie die damit verbundenen Implikationen aufgreift. Esping-Andersen unterscheidet zwischen: a) dem sozialdemokratischen Modell, das sich auf universelle, an soziale Bürgerrechte gebundene Ansprüche auf Sozialleistungen stützt, sich eher durch allgemeine Besteuerung als durch ein Versorgungssystem auf Versicherungsbasis finanziert und sich zu weitreichender egalitärer Redistribution und hohen Standards öffentlicher Fürsorgeleistungen verpflichtet; b) dem konservativen Modell, in dem unterschiedliche Ansprüche auf Sozialleistungen an Beschäftigung und sozialen Status gekoppelt werden, Sozialleistungen auf einem Versicherungssystem basieren und an korporativistische Organisationen gebunden sind. Sozialpolitik hat in diesem System keine egalitären Ziele, selbst wenn sie bis- 
weilen umfangreiche staațliche Fürsorgeleistungen beinhalten kann. Im c) liberalen Modell werden Einzelpersonen begrenzte Ansprüche auf Sozialleistungen zugestanden und/oder Einzelleistungen bei nachzuweisender Bedürftigkeit. Die Finanzierung stützt sich auf individuelle Versicherungen bzw. bei Bedürftigkeit auf allgemeine Besteuerung. Die gesellschaftliche Reproduktion ist dabei maßgeblich an private, vom Markt bereitgestellte Versorgung gebunden, und zur sozialen Sicherung existiert nur ein weitmaschiges Netz, für das nur sehr begrenzt staatliche Mittel eingesetzt werden. Das sozialdemokratische Modell ist mit einer festen, politisch geplanten und institutionell gestützten politischen Verpflichtung zur Vollbeschäftigung verbunden, die ein hohes Maß an politischer Mobilisierung von seiten der organisierten Arbeiterklasse und ihrer Verbündeten unter den Bauern und/oder den neuen Mittelklassen erfordert. Den liberalen Wohlfahrtsstaaten mangelt es hingegen an einer langfristigen politischen Basis und/oder an adäquaten institutionellen Mechanismen zum Erreichen der Vollbeschäftigung, auch wenn sie sich formal zur Absicherung der Vollbeschäftigung verpflichten. Darüber hinaus liegt ihre Priorität bei anderen ökonomischen und politischen Zielen. Wohlfahrtsstaaten mit einer konservativen Tradition bilden unter dem Gesichtspunkt der Vollbeschäftigungsverpflichtung keine einheitliche Gruppe. Hierbei hängt viel von der Art der Abgrenzung zu linker politischer Mobilisierung und von der Stärke christlich-sozialer Massenparteien gegenüber einer Wohlfahrtspolitik ab, die die Prinzipien des freien Marktes herausfordert (Esping-Andersen 1986, S. 226-34, 243 f.). Klassenkräfteverhältnisse sind nicht nur historisch für die Entwicklung dieser Wohlfahrtsstaatmodelle bedeutend, sondern wirken dauerhaft. Historisch setzte sich das konservative Modell vorwiegend in jenen Staaten durch, in denen die Kirche bei der Sozialreform eine zentrale Rolle spielte, der Absolutismus stark war und nur langsam verschwand. Deswegen ist es vor allem in den Staaten anzutreffen, in denen die bürgerliche Revolution schwach und unvollständig war oder völlig ausblieb, wie in Österreich, Deutschland, Frankreich, Italien, Japan und Belgien. Liberale sozialpolitische Regime entwickelten sich hingegen in Staaten, wo der bürgerliche Impuls besonders stark war. Neben Großbritannien lassen sich die Länder der »Neuen. Welt« wie die Vereinigten Staaten, Kanada und Australien anführen. Das sozialdemokratische Modell schließlich ist in jenen Ländern vorherrschend, in denen die Arbeiterbewegung lange Zeit relativ geeint und stark mobilisiert war, dauerhafte Bündnisse mit anderen Klassen geschlossen hatte und sich einer schwachen und/oder zersplitterten Bourgeoisie gegenübersah. Die herausragendsten Beispiele für dieses Modell sind Schweden und Norwegen (vgl. Esping-Andersen 1986; Flora 1985). Überdies entwickeln sozialpolitische Herrschaftsformen, wenn sie sich erst einmal etabliert haben, ein institutionelles Eigengewicht: sie legen den Rahmen für weitere sozialpolitische Entwicklungen fest, verändern das Kräftegleichgewicht und institutionalisieren Gruppeninteressen sowohl bei den Wohlfahrtsempfängern wie auch bei den »Produzenten« sozialer Sicherheit.

\section{Der differenzierte und der integrierte Woblfahrtsstaat}

Mishra unterscheidet zwischen dem integrierten und dem differenzierten Wohlfahrtsstaat. Dies bezieht sich auf unterschiedliche Verknüpfungen der wirtschafts- und der sozialpolitischen Seite des modernen Interventionsstaates. Ersterer integriert Vollbeschäftigungs- und Sozial politik durch eine Koordinierung von Nachfrage- und Angebotsmanagement, von kapitalistischer Investitionspolitik und Arbeitskräfteangebot, von ökonomischer Steuerung und Sozialpolitik. Mishra fügt hinzu, daß dieses Modell auch mit einer produktivistischen 
Rechtfertigung des kollektiven Konsums vom »Humankapitak-Standpunkt aus verbunden wird und üblicherweise von zentralisierten Verhandlungen zwischen den organisierten Sozialpartnern begleitet ist. Er stellt diesem Modell den differenzierten Wohlfahrtsstaat entgegen, in dem Wirtschafts- und Sozialpolitik getrennt sind. Dabei wird die Vollbeschäftigungspolitik zum großen Teil durch makroökonomisches Nachfragemanagement betrieben. Staatliche Wohlfahrtspflege nimmt dabei wirtschaftliche Entwicklungen tendenziell als gegeben hin und muß auf sie reagieren bzw. sich ihnen anpassen. Sozialpolitik wird hier als eigene politische Arena gesehen, in der sich eine Vielzahl von Interessengruppen spezifische soziale Rechte, Dienstleistungen und Transferzahlungen aushandeln (Mishra 1985; zum Primat der Wirtschaftspolitik und der wirtschaftspolitischen Arena Walker 1983).

Bis zu einem gewissen Grad überlappen sich diese Typologien, denn der liberale Wohlfahrtsstaat ist in seiner typischen Form ein differenzierter Wohlfahrtsstaat. Das heißt jedoch nicht, daß der integrierte Wohlfahrtsstaat nur ein sozialdemokratischer sein kann. Denn obwohl dieser gewöhnlich seine Wirtschafts- und Sozialpolitik auf das Ziel der Vollbeschäftigung ausrichtet, bestehen ähnliche Tendenzen auch in Gesellschaften, die in der Tradition konservativer Wohlfahrtsmodelle stehen. Während Mishra also Österreich als den Archetypus des integrierten Wohlfahrtsstaats darstellt, verweist Esping-Andersen auf die Tradition des konservativen Wohlfahrtsmodells. Spezifische institutionelle Strukturen und Veränderungen im politischen Kräfteverhältnis haben für die Herausbildung der augenblicklichen ökonomischen und suzialpolitischen Orientierung von Gesellschaften mit einer konservativen Wohlfahrtstradition eine wichtige Rolle gespielt. Unter dem Einfluß von entsprechenden strukturellen und strategischen Faktoren können sich solche Gesellschaften entweder in Richtung eines sozialdemokratischen integrierten oder eines liberalen differenzierten Modells des Wohlfahrtsstaates entwickeln. Diese Tendenzen müssen jedoch auch im Zusammenhang mit dem fordistischen Kontext gesehen werden, in dem sich die Wohlfahrtsstaaten der Nachkriegszeit entwickelt haben. Denn wie wir weiter unten erläutern werden, enthält der Übergang zum Postfordismus die Bedingungen für einen integrierten liberalen Wohlfahrtsstaat. Dieser könnte eine flexible Angebotspolitik und Maßnahmen zur sozialen Sicherheit in einer Weise verbinden, die mehr im Interesse des Kapitals als in dem der Arbeit liegt, und deshalb ein Klassenkräfteverhältnis bedingt, das von jenem erheblich differiert, das den sozialdemokratischen Wohlfahrtsstaat getragen hat.

\section{Akkumulationsregime}

Wir müssen nun die Verbindung zwischen der Krise im Wohlfahrtsstaat mit der Krise des Fordismus und dem Übergang zum Postfordismus herstellen. Fordismus und Postfordismus sind Akkumulationsregime und Regulationsweisen. Ein Akkumulationsregime zeichnet sich durch eine kontingente, historisch konstituierte und gesellschaftlich reproduzierte Korrespondenz von Produktions- und Konsummustern aus. Seine grundlegenden Merkmale sind: verschiedene Bedingungen der Arbeitskräftenutzung, die Merkmale des Lohnverhältnisses, die Investitionsdynamik, die Wettbewerbsformen und das Geld- und Kreditsystem (Mazier et al. 1985, S. 9). Eine Regulationsweise beinhaltet »die Gesamtheit der institutionellen Formen, Netze und expliziten oder impliziten Normen, die die Vereinbarkeit von Verhaltensweisen im Rahmen eines Akkumulationsregimes sichern, und zwar sowohl entsprechend dem Zustand der gesellschaftlichen Verhältnisse als auch über deren konfliktuellen Eigen- 
schaften hinaus (Lipietz 1985, S. 121; zu "gesellschaftlichen Akkumulationsstrukturen« vgl. Bowles und Edwards 1985). Die Strukturen des Staates und der Sozialpolitik gehören zu den wichtigsten Aspekten einer Regulationsweise.

In diesem Kontext werden zwei zentrale Formen von Akkumulationsregimes unterschieden: die extensive (oder kompetitive) und die intensive (oder monopolistische). Das extensive Regime beruht auf Profitsteigerung durch Verlängerung und Verdichtung des Arbeitstages, sowie der Ausweitung des Kapitalverhältnisses auf weitere Produktionsbereiche. Ein intensives Regime basiert auf der Produktion relativen Mehrwerts, d.h. auf Produktivitätssteigerungen durch die Einführung neuer Produktionstechniken, so daß mehr produziert wird bei gleichbleibender Länge des Arbeitstages und bei gleicher Arbeitsintensität. Hier werden wir nur auf die Form eingehen, die das intensive Akkumulationsregime mit dem Wiederaufschwung nach der »Großen Depression « in den 30er Jahren angenommen hat. Diese Form wird generell als Fordismus bezeichnet.

\section{A. Der Fordismus}

Der Fordismus ist, in wenigen Worten, ein auf Massenproduktion und Massenkonsum basierendes Modell der Kapitalakkumulation. In den letzten Jahren ist viel über den Fordismus geschrieben worden, und wir wollen hier auf die anwachsende Literatur nicht weiter eingehen. Es soll jedoch auf zwei verschiedene Problemfelder hingewiesen werden, die in diesen Untersuchungen aufgezeigt werden können. Das erste Problemfeld betrifft die Bedeutung, die dem Arbeitsprozeß im Verhältnis zu allgemeinen gesellschaftlichen Akkumulationsstrukturen und Vergesellschaftungsmustern beigemessen wird. Das zweite Problemfeld betrifft die Bedeutung von nationalen und innerstaatlichen Regulationsweisen im Verhältnis zu den internationalen Aspekten der fordistischen Expansion (wie die US-Hegemonie, der Ausgleich zwischen den unterschiedlichen staatlichen Regulationweisen und die Merkmale des peripheren Fordismus). Ein sorgfältiger Ansatz müßte alle vier Aspekte des Fordismus betrachten. Da unser unmittelbares Thema jedoch der Wohlfahrtsstaat ist, wird sich dieser Aufsatz auf die Rolle der Sozialpolitik in nationalen Regulationsweisen konzentrieren.

In diesem Kontext können einige allgemeine Merkmale des Fordismus in den fortgeschrittenen kapitalistischen Gesellschaften, in denen sich der Wohlfahrtsstaat entwickelt hat, skizziert werden. ${ }^{7}$ Dabei sind zehn Elemente besonders wichtig:

1. die Entwicklung der Massenproduktion - besonders auf dem Konsumgütersektor, aber auch in manchen Bereichen der Investitionsgüterbranche -, die auf spezialisierter Großserienproduktion basiert (oft mit der Mechanisierung der Transferaktivitäten durch Techniken wie Fließband oder kontinuierliche Fließprozesse gekoppelt);

2. die vorrangige Verwendung von maschinenbestimmter angelernter Arbeit anstelle von handwerklich ausgebildeter und nicht-taylorisierter, unqualifizierter Arbeit;

3. die betriebswirtschaftliche Orientierung auf die wissenschaftliche Organisation des gesamten kollektiven Arbeitsprozesses in einem Unternehmen statt auf fragmentierte, vom unqualifizierten Einzelarbeiter erbrachte Arbeit (d.h. die Dominanz des Fordismus über den Taylorismus);

4. die Rolle des Lohns (im Gegensatz zu häuslicher Arbeit, Subsistenzlandwirtschaft oder Arbeitsmigration) als zentraler Mechanismus und/oder Bezugspunkt bei der Sicherstellung der Reproduktion der Arbeitskraft; 
5. die Konsolidierung von kollektiven Tarifverhandlungen über Lohnhöhe (abhängig von Inflation und/oder Produktivitätssteigerungen) und die Arbeitszeit mit dem Ergebnis, daß Unternehmen besser in der Lage sind, Lohnkosten und Konsumentennachfrage im Voraus zu berechnen;

6. die Vorherrschaft des Massenkonsums von standardisierten, in Großserien hergestellten Gütern und/oder des kollektiven Konsums staatlich bereitgestellter Güter und Dienstleistungen im Gegensatz zu nicht-standardisierten, gewöhnlich handwerklich gefertigten oder eigenproduzierten Gütern und Dienstleistungen;

7. die Rolle der marginalen Produktdifferenzierung (z.B. jährliche Modellveränderungen), von eingebauter Obsoleszenz und Werbung zur Stimulierung der Massennachfrage sowie eine zunehmende Rolle von Konsumentenkrediten bei der Finanzierung von Massenkonsum;

8. die zentrale Rolle des Massenkonsums für den Kapitalkreislauf, in dem sich die Ausweitung des Kapital- und des Konsumgütersektors wechselseitig verstärkt;

9. die zunehmende Bedeutung des Staates bei der Sicherung der Rahmenbedingungen für die Kapitalakkumulation und die Reproduktion der Lohnarbeit durch Anpassung der Märkte an die Starrheiten der fordistischen Massenproduktion über eine staatliche Lohn-, Arbeitsmarkt- und Nachfragepolitik;

10. die Entwicklung des Wohlfahrtsstaates als Mechanismus zur Einrichtung eines sozialen Mindestlohns, für eine Verallgemeinerung der Massenkonsumnormen und zur Koordinierung von Kapital- und Konsumgütersektoren.

Die letztgenannten drei Punkte sind für eine Analyse des Wohlfahrtsstaates besonders zentral. Erst mit dem Übergang zum Fordismus wurde nämlich die ökonomische und soziale Basis des Wohlfahrtsstaates im eigentlichen Sinne (wie oben definiert) sichergestellt. Denn erst die Entwicklung eines Akkumulationsregimes, das sich auf eine Verbindung von Massenproduktion mit Massenkonsum stützte, versetzte den Staat in die Lage, die Interessen von Kapital und Arbeit durch ein Programm von Vollbeschäftigung und sozialer Wohlfahrt zu vermitteln.

\section{B. Die Krise des Fordismus}

Die Krise des Fordismus manifestiert sich in verschiedenen Bereichen: im Arbeitsprozeß, im Lohnverhältnis, in der Rolle des Staates, im internationalen Geldsystem, in der US-Hegemonie usw. Diese vielfältige Krise spiegelt sich in verschiedenen (und oft divergierenden) Antworten auf verschiedenen Ebenen wider, die vermutlich erst auf eine neue Weise verknüpft werden müssen, wenn es zu einer neuen langen Welle des Akkumulationsprozesses kommen soll.

Der Zusammenbruch der tayloristischen und fordistischen Arbeitsorganisation kann auf zwei Rigiditäten zurückgeführt werden. Zum einen sind die Einführung starrer Produktionstechnologien und der Realisierung damit verbundener Skalenerträge technische Grenzen gesetzt. Nicht alle Produktionszweige eignen sich für die Anwendung von tayloristischen und fordistischen Methoden, und der Umfang weiterer Produktivitätssteigerungen erschöpfte sich recht bald, als diese Techniken erst einmal so weit wie möglich verallgemeinert worden waren. Zum zweiten hatte die Arbeiterklasse dem tayloristischen und fordistischen Produktionsprozeß wachsenden Widerstand entgegengesetzt, der sich auch auf andere Wirtschaftssektoren ausweitete. 
Der Fordismus bringt gewisse Rigiditäten in der Produktionsorganisation mit sich, die durch die Dominanz der an bestimmte Produkte ${ }^{8}$ gekoppelten Produktionsstrukturen, und im weiteren durch die Anbindung des zirkulierenden Kapitals an diese Produktionsstrukturen bedingt sind. Auf Unternehmensebene gab es zwar bestimmte Flexibilitätsreserven (z.B. durch variable Lagerhaltung, Kurzarbeit oder Überstunden, Inanspruchnahme von Krediten etc.), und wo es eine duale Ökonomie gab, konnten Anpassungskosten Subunternehmern und nichtorganisierten Arbeitern aufgeladen werden. Diese Mikroformen der Flexibilität waren jedoch begrenzt, und sie sind dort am effektivsten, wo der keynesianische Staat eine Anpassung auf der Makroebene sicherstellt. Das keynesianische Nachfragemanagement schien ökonomische Fluktuationen dämpfen und ein stabiles, kalkulierbares Wachstum sichern zu können. Hochspezialisierten Unternehmen sicherte dies steigende Erträge und ermunterte sie zu Investitionen (vgl. Galbraith 1967; Kundig 1984; Boyer und Coriat 1986).

Als der Nachkriegsboom nachließ, erwiesen sich diese begrenzten Flexibilitäten jedoch als unzureichend. Die Löhne stiegen schneller als die Produktivität, und auch der Wohlfahrtsstaat expandierte. Dies übte einen zweifachen Druck auf die Profite aus, und in dem Maße, in dem die Ausgaben des Wohlfahrtsstaates durch Lohn- und Einkommens- und/oder Verbrauchssteuern und/oder durch Inflation finanziert wurden, waren auch die Arbeiter ökonomischem Druck ausgesetzt. Die Vollbeschäftigungsverpflichtung bedeutete auch, daß die Wirkungen von "Reservearmeen" auf sekundäre Märkte begrenzt blieben, und dies verzögerte oder stoppte die Verbesserung der Profitmöglichkeiten während der konjunkturellen Abschwünge (Boyer und Coriat 1986). Zum zweiten kam es im Zusammenhang mit dem verstärkten Widerstand der Arbeiterklasse zu anwachsendem Absentismus und Fluktuation, vermehrter Ausschußproduktion und einer Zunahme von oft örtlich begrenzten Streiks mit gravierenden Rückwirkungen auf den Produktionsfluß und mit einer Ausweitung von Auseinandersetzungen und Streiks um Themen, die sich nicht in die abstrakten, universellen Kategorien von Geld und Zeit einpassen ließen (Crouch und Pizzorno 1978; Mazier et al. 1985, S. 32-33; Hirsch 1985a). Im Gegenzug führte dies zu einer Suche nach neuen Formen der kapitalistischen Produktionsorganisation, die die Starrheiten des. Taylorismus und des Fordismus überwinden und dem Widerstand der Arbeiterklasse et was entgegensetzen könnten. Auf die Arbeiterklasse bezogen, waren die Antworten zunächst innerhalb fordistischer Muster angesiedelt und beinhalteten Job enrichment, Entwicklung autonomer Gruppen innerhalb eines unveränderten Fließbandsystems und bürokratischer Kontrollen. In letzter Zeit läßt sich eine Bewegung in Richtung postfordistischer Antworten verzeichnen, die auf eine größere Flexibilisierung der Produktion zielen, welche durch die Ausweitung von Automation und Roboterisierung möglich wird und die mit Versuchen verknüpft ist, die Arbeiterschaft neu zusammenzusetzen (Hirsch 1985a; Kundig 1984; Mazier et al. 1985, S: 294; Morville 1985). Diese Aspekte der Krise hängen eng miteinander zusammen. Mehrere Studien deuten an, daß die fordistische Akkumulation von einer spezifischen Balance zwischen Profiten und Löhnen abhängt, die durch die Akrivitäten von Gewerkschaften und Staat sichergestellt werden muß. Vor allem Boyer und Coriat haben gezeigt, daß der Lohnindex im Verhältnis zu den Erträgen, zur Konsumneigung und den Investitions- und Nachfragerelationen weder zu hoch noch zu niedrig sein darf (Boyer und Coriat 1986; vgl. Bowles und Edwards 1985; Przeworski 1985; und Lipietz 1985). Wenn er zu hoch liegt, sinken Profite und Investitionen. Liegt er zu tief, ist die Massennachfrage zu schwach, um Invest itionsanreize zu liefern. Der Fordismus gedeiht dort am besten, wo sich Unternehmen zu einer oligopolistischen 
Preispolitik verpflichten (durch die die Investitionen finanziert werden) und die Löhne an Produktivitätszuwächse unterhalb der Erträge durch Neuinvestitionen gebunden werden (so daß auch der Massenkonsum steigt) (Boyer und Coriat 1986; Hurtienne 1986). Als es mit reduzierten Erträgen, geringerer Produktivität und gleichzeitig mit Löhnen konfrontiert wurde, die auf der Basis vergangener Profit - und Produktivitätsniveaus ausgehandelt worden waren, begann das fordistische System Symptome der Stagnation zu zeigen.

\section{Postfordismus}

Die Krise des Fordismus umfaßt mehr als nur die der Produktivkräfte oder der Profitabilität im einfachen Sinn. Viel grundlegender ist die Unfähigkeit des Kapitals, ein neues Akkumulationsregime zu schaffen, mit angemessenen institutionellen Formen, sozialen Beziehungen und einem Kräftegleichgewicht innerhalb des Machtblocks und in der Bevölkerung. Nur wenn ein neuer »historischer Block « (um Gramscis Begriff von der nicht notwendigen Korrespondenz zwischen Basis und Überbau zu benutzen) aufgebaut werden kann, wird die Kapitalakkumulation (mit dem Einsatz alter und neuer Technologien) eine weitere lange Welle der Expansion erleben (Hirsch und Roth 1986; Mazier et al. 1985, S. 295). In diesem Sinn muß der qualitativ neue Charakter des Postfordismus nicht nur in Veränderungen der Produktionsorganisation (am offensichtlichsten bei neuen Formen der Flexibilisierung) gesucht werden, sondern auch in der zunehmenden Bedeutung des Staates und der allgemeinen Reorganisation von Vergesellschaftungsformen (vgl. Boyer und Coriat 1986; Hirsch 1985b; Jessop 1983, 1986b; Mazier et al. 1985; Lipietz 1985).

Die Einzelheiten des »postfordistischen « Systems sind noch unklar, und es wird offenbar einige Merkmale des Fordismus übernehmen. Aber zwei Hauptunterschiede betreffen die Reorganisation der Produktion und die Neuzusammensetzung der Arbeitskräfte. Wir behandeln zunächst die Reorganisation der Produktion.

Mit dem Postfordismus tritt eine Grundtendenz zur flexiblen Spezialisierung in zuvor rigide standardisierten Produktionsprozessen und/oder im Bereich der Kleinserienproduktion auf, die zuvor fordistischen Praktiken widerstanden hatten. Zusätzlich gibt es einen neuen Trend zu flexibler Automatisierung, der nicht nur neue Produktionsprozesse, sondern auch neue Produkte umfaßt. Flexible Spezialisierung ermöglicht die Serienproduktion einer Vielzahl von Produkten durch flexible Fertigungssysteme. Das hat vorteilhafte Kurzzeiteffekte auf die Wettbewerbsposition von Avantgarde-Firmen: nachhinkende Firmen fallen aus dem Markt, die Kosten werden reduziert, der Ausstoß kann gesteigert werden. Wenn sich aber die flexible Spezialisierung in einem Sektor allgemein durchgesetzt hat, wird jedoch durch den fortgesetzten Preisverfall keine zusätzliche Nachfrage nach flexibel produzierten Waren geschaffen, es sei denn, die traditionellen Konsumnormen ändern sich ebenfalls. Flexible Spezialisierung stellt daher ein Mittel zur Anpassung an langsameres Wachstum dar, mittels Produktdifferenzierung und Marktsegmentierung auf kompetitiven, aber stagnierenden Märkten. Im Gegensatz dazu hat die flexible Automatisierung in der Produktion neuer Massenkonsumgüter ein langfristiges Potential. Die Preise fallen, die Produktivität wächst, die Löhne können ebenfalls steigen und die Verbreitung der neuen Technologien kann die Profite selbst in älteren Industrien steigern, die einst auf der Fließbandfertigung basierten. Beide Formen der Flexibilisierung sind eng mit der zunehmenden Bedeutung von Elektronik, Mikroprozessoren und Informationstechnologien bei der Produktion von Waren und Dienstleistungen verbunden. Jedwede neue lange Welle der Akkumulation muß auf der flexiblen 
Automatisierung und nicht nur auf flexibler Spezialisierung aufbauen (Boyer und Coriat 1986; Mazier u.a. 1985).

Aber Flexibilisierung ist auch mit Veränderungen in der Betriebsorganisation und der Beschäftigungsstruktur verknüpft. Insbesondere fällt eine verstärkte Polarisierung der Arbeitskräfte in einen vollbeschäftigten, ausgebildeten Kern und einen ungelernten Rand auf, die oft nur teilzeitbeschäftigt ist. Die Randbelegschaften sind oft neuen Formen taylorisierter Arbeit unterworfen, mobiler und flexibler, stärker zersplittert und individualisierter (Hirsch 1985b, S. 11). Der vollbeschäftigte Kern hat oft geistige und/oder Überwachungsfunktionen übernommen, und muß auf jeden Fall im Einsatz von Fertigkeiten flexibel sein. Insgesamt kontrastiert diese Polarisierung scharf mit den homogenisierenden Tendenzen, die mit dem Aufstieg des »wohlhabenden «, gutbezahlten Massenarbeiters unter dem Fordismus einhergingen. Sie erstreckt sich über die Bereiche flexibler Fabrikation hinaus und breitet sich auch im tertiären bzw. Dienstleistungssektor aus. Hier ist die Einführung flexibler Text- und Datenverarbeitungssysteme von einer flexibleren Arbeitsorganisation begleitet, die ebenso Heim-, wie Teilzeit- und Schichtarbeit umfaßt.

Es ist unwahrscheinlich, daß die flexible Spezialisierung (im Gegensatz zur flexiblen Automatisierung) zusätzliche Nachfrage schaffen oder eine Rückkehr zur Vollbeschäftigung sichern wird. Während der Fordismus eine Politik der Vollbeschäftigung und Sozialrechte zur Nachfragesicherung förderte, und dadurch die Grundlage für einen Klassenkompromiß zwischen Kapital und Arbeit schuf (vgl. Przeworski 1985), stellt das neue postfordistische Regime für Vollbeschäftigung und darauf abgest ellte Klassenbündnisse ein ernsthaftes Problem dar. Dies hat große Auswirkungen auf die Rolle der Gewerkschaften und des Staates und erfordert neue Klassenallianzen. Die Polarisierung und Zersplitterung der Belegschaften muß nicht die Rolle der Gewerkschaften auf Fabrik- und Unternehmensebene schmälern. Aber die Entwicklungen, die mit dem Postfordismus verbunden sind, könnten die Bedeutung der Gewerkschaftsbewegung als Sozialpartner in einem Vollbeschäftigungspakt schwächen. Zu rechnen ist insbesondere mit einem niedrigeren Grad gewerkschaftlicher Organisierung, der Dezentralisierung und Zersplitterung nationaler Gewerkschaftsorganisationen und dem Entstehen einer - verglichen mit dem Aufkommen des Massenarbeiters unter dem Fordismus - polarisierten Beschäftigungsstruktur. Auf der anderen Seite wird dies mit einer reduzierten Rolle des Staates bei der Sicherung von Vollbeschäftigung einhergehen (obgleich seine Rolle bei der Regelung des Lobnverbältnisses erhalten bleiben wird). In der Tat sind Mazier u.a. der Ansicht, das wichtigere Problem für den Staat werde es sein, die Gesamtheit der sozialen Ausgaben zu bewältigen (Gesundheit, Bildung, etc.), die die Lohnnebenkosten erhöhen. Sie sehen das Risiko bei der Einführung neuer Technologien in der Steigerung der Produktivität ohne einen entsprechenden Abbau der Arbeitslosigkeit oder der sozialen Kosten (Mazier u.a., 1985, S. 297). Die Reorganisation des Sozialstaates wird daher zu einem wesentlichen Bestandteil beim Übergang zum Postfordismus.

\section{Postfordismus und Staat}

Für den Beginn einer neuen langen Welle braucht es wesentlich mehr als neue Produktivkräfte und einen reorganisierten Arbeitsprozeß. Veränderungen in der Regulationsweise (oder der gesellschaftlichen Akkumulationsstruktur) sind ebenso erforderlich. Das politische System und die allgemeinen Vergesellschaftungsformen müssen neu formiert werden. In die- 
sem Zusammenhang müssen wir bei der Periodisierung der veränderten Staatsfunktionen vorsichtig sein, denn die Rolle des Staates in der gegenwärtigen Übergangsphase wird sicherlich von der in einem konsolidierten postfordistischen System verschieden sein. ${ }^{9}$ Oft wird behauptet, der Staat sei bei der Errichtung der Rahmenbedingungen für den Aufstieg des Kapitalismus aktiv gewesen, zurückhaltend während der Phase des liberalen Konkurrenzkapitalismus und interveniere jetzt unter dem Monopolkapitalismus aufs Neue. Es wird jedoch seltener berücksichtigt, daß ähnliche Phasen des (Nicht-)Eingreifens in jedem Stadium der kapitalistischen Ent wicklung anzutreffen sind. So war staatliche Politik an der Schaffung der Bedingungen für den Nachkriegsboom zur Zeit des Wiederaufbaus aktiv beteiligt, zur Hochzeit des Nachkriegsbooms in gewisser Weise nicht engagiert, und sie begann intensiver zu intervenieren, als der Boom zu stocken anfing, um das fordistische System aufrechtzuerhalten. Heutzutage muß der Staat abermals auf dem Angebotssektor intervenieren, um die Rahmenbedingungen für eine postfordistische lange Welle der Kapitalakkumulation zu schaffen. Dies ist selbst unter vorgeblich liberalen Regierungen offensichtlich, wie auch bei interventionsorientierten Regime. Somit zeichnet sich die gegenwärtige politische Konjunktur dadurch aus, daß der Staat sich gleichzeitig zurückzieht (von seiner stützenden Rolle in der Niedergangsphase des Fordismus) und eingreift (um die Entstehung der nächsten langen Welle zu fördern). Das bringt größere Widersprüche für seine Politik, Programme und Legitimation mit sich.

Diesen Punkt gilt es aufgrund von zwei kontrastierenden (aber sich ergänzenden) Irrtümern in der Diskussion der Rolle des Staates im Nachkriegsboom und in der gegenwärtigen Lage zu unterstreichen. Die Anhänger der gegenwärtigen Regierungspolitik in Großbritannien und anderswo vertreten die Ansicht, ein stärker interventionistischer Staat sei zurückgestutzt worden und der Staat spiele nunmehr eine liberalere laissez-faire Rolle. Diese Argumentation verwechselt die reale Rolle des Staates am Ende der Nachkriegsphase mit seiner antizipierten beschränkteren Bedeutung auf dem Höhepunkt der kommenden Phase. Umgekehrt sind Kritiker dieser Politik der Ansicht, die Rolle des Staates im Nachkriegsboom habe wenig mehr umfaßt als die Nachfrageregulation auf der Makroebene und die Versorgung mit öffentlichen Sozialleistungen und kontrastieren dies mit den gegenwärtigen Trends zu wachsender Intervention auf der Angebotsseite, größeren Veränderungen im Sozialstaat und einem stärkeren und aktiveren »law and order «-Staat. Dieser Vergleich unterstreicht die begrenzte Rolle des Staates auf dem Höhepunkt des Nachkriegsbooms und vernachlässigt seine wichtigen Angebotsfunktionen während der Anfangsphase des Wiederaufbaus nach dem Kriege. Gerade diese letzteren Funktionen sind voll vergleichbar mit jenen, die gegenwärtig wahrgenommen werden, um die Bedingungen für eine neue Phase der Akkumulation zu sichern. So vergleichen weder Anwälte noch Kritiker der gegenwärtigen Regierungspolitik gleiches mit gleichem, und der Charakter der Staatsintervention wird deshalb falsch verstanden.

\section{Postfordismus und Wohlfahrtsstaat}

Während der fordistischen Periode hat der Staat in den fortgeschrittenen kapitalistischen Ökonomien eine Schlüsselrolle bei der Integration von Investitions- und Konsumgüterindustrien ${ }^{10}$ und einer darauf abgestimmten Regulierung des Lohnsystems gespielt. Vollbeschäftigung wird oft als Hauptziel der Politik während dieser Periode angeführt, aber ihr Erreichen war tatsächlich viel mehr auf die Grunddynamik der fordistischen Expansion gegründet als 
auf die Feinabstimmung durch die Beschäftigungspolitik der Regierung. Der wirkliche Test für diese politische Zielbestimmung kam mit dem Zusammenbruch des Nachkriegsbooms, der aufkommenden Krise des Fordismus und solch kontingenten Ereignissen wie dem sogenannten »Ölpreisschock«. Der offenbare Erfolg des Sozialstaates war ebenfalls in dreierlei Hinsicht auf den Nachkriegsboom gegründet. Erstens brachte der fordistische Aufschwung die Steuereinnahmen zur Finanzierung der Ausdehnung des Wohlfahrtsstaates. Zweitens reduzierte die Vollbeschäftigung in dem Maße, wie sie auf einem Arbeitsmarkt erreicht wurde, der eher einheitlich denn segmentiert war, den Umfang grundlegender Armut bei Arbeiterfamilien. Dies wiederum schuf den Raum für großzügigere Programme zur Einkommenssicherung für andere Gruppen (und verallgemeinerte dadurch die Normen des Massenkonsums) und/oder für die Ausweitung der Sozialleistungen auf andere Bereiche (oft verbunden mit neuen Anforderungen an die soziale Reproduktion, die der Fordismus mit sich brachte). Und drittens schuf oder intensivierte die mit dem Fordismus verbundene Vergesellschaftungsform »soziale Probleme«, für die wohlfahrtsstaatliche Lösungen gesucht werden konnten. " Aber die Ausdehnung des Wohlfahrtsstaates ging so weit, daß sie einige der Bedingungen untergrub, die das fordistische Akkumulationsregime stützten. Denn sie verschob das Klassenkräfteverhältnis im ökonomischen Bereich zugunsten der organisierten Arbeit - eine Verschiebung, die erst kritisch wurde, als die Krise des Fordismus in Erscheinung trat und das Kapital versuchte, den Arbeitsprozeß neu zu strukturieren und die Lohnkosten zu senken. Der Fordismus institutionalisierte einen Soziallohn, dessen Starrheit nach unten (stärker als seine Aufwärtstendenz) als Akkumulationsbremse wirken konnte. Zusätzlich entwickelte der Wohlfahrtsstaat eine eigene Wachstumsdynamik mit schwerwiegenden Folgen für Struktur und Ressourcen des fordistischen Akkumulationsregimes. So erlebte der Wohlfahrtsstaat neben dem offensichtlichen Anstieg des Budgets für Sozialausgaben (mit seinen Folgen für die Umstrukturierung des Steuer- und Kreditsystems) Veränderungen seiner grundlegenden strukturellen Formen, der damit verbundenen sozialpolitischen Arenen und der dort wirksamen politischen Träger. Diese Veränderungen bedrohten das fordistische Modell durch ihren Einfluß auf beide Seiten des Verhältnisses von Kapital und Arbeit (hinsichtlich des Kräfteverhältnisses wie auch bezogen auf den Anreiz, zu investieren und/oder zu arbeiten) und auf die grundlegenden Vergesellschaftungsmuster (besonders hinsichtlich der relativen Abkopplung der Sozialpolitik vom Kapitalkreislauf). In diesem Sinne kann man die Krise des Wohlfahrtsstaates als Gelegenheit für das Kapital interpretieren, im Interesse einer erneuerten Akkumulation gewaltsam die Einheit von Wirtschafts- und Sozialpolitik erneut durchzusetzen.

\section{A. Die Krise des fordistischen Woblfabrtsstaates}

Die gegenwärtige Krise des Wohlfahrtsstaates ist ein komplexes, vielseitiges und überdeterminiertes Phänomen. Wir wollen uns hier auf ihre Grundlegung in der Wechselbeziehung zwischen fordistischem Akkumulationsprozeß und der Eigendynamik des Wohlfahrtsstaates konzentrieren. Diese Prozesse stützten sich während der Boomjahre des Fordismus gegenseitig, doch sie beeinflußten einander während der Krisenjahre negativ. Wir behandeln zunächst die ökonomischen und dann die politischen Aspekte der Krise.

\section{1. Ökonomische Faktoren}

Die Krise des Fordismus hat auf die Finanzen des Wohlfahrtsstates offensichtlich einen »Scheren«-Effekt ausgeübt. Auf der Einkommensseite hat sie die Steuerbasis für Soziallei- 
stungen in dem Maße reduziert, wie diese an Steuern auf Löhne und Gehälter gebunden ist. Der Beitrag des Kapitals zu den staatlichen Einkünften wird ebenso durch den Rückgang der Bruttoprofite wie durch die Umverteilung der Steuerlasten zum Schutz des Niveaus der Profite nach Steuern reduziert. Gleichzeitig hat die Krise den Ausgabenbedarf zur Einkommenssicherung (z.B. Arbeitslosengeld, Vorruhestandsleistungen, Familienhilfen) und mit den Auswirkungen von Arbeitslosigkeit und Rezession den Bedarf an anderen sozialen Leistungen (wie Wohnungs-, Gesundheits- und Familienprogramme) gesteigert. Wo der Staat überdies seine realen und/oder Steuerausgaben für technologische Innovation und strukturelle Reorganisation gesteigert, und/oder allgemein die Kapitalsteuern gesenkt hat, wurden die für Sozialausgaben verfügbaren Ressourcen weiter eingeschränkt. ${ }^{12}$ Die daraus resultierende allgemeine Finanzkrise des Staates ist mit Konflikten nicht nur über Höhe und Verteilung von Staatsausgaben, sondern auch über die Umstrukturierung des Steuer- und Kreditsystems verbunden. Dies spiegelt sich in der wachsenden Ablehnung der durch den Wohlfahrtsstaat bedingten Steuerlasten und/oder der inflatorischen Folgen der Sozialausgaben bei staatlicher Kreditaufnahme wider. Doch die Möglichkeiten für eine langfristige Kürzung der Sozialausgaben (besonders zugunsten des Kapitals) sind begrenzt: allenfalls könnte eine Umgewich. tung von öffentlichen auf private Sozialleistungen stattfinden. Gleichzeitig wuchs der Widerstand gegen die sozialen und wirtschaftlichen Auswirkungen der Kürzungen im Sozialbudget (besonders in den Bereichen Gesundheit, Bildung und Renten), sobald sie über praktische Randbereiche hinausgingen und/oder eher soziale Kern-als Randgruppen betrafen.

Wenn man sich nur auf die Finanz- und Haushaltsaspekte des Wohlfahrtsstaates konzentriert, erfaßt man lediglich zwei Zentralbereiche seiner wirtschaftlichen Krise. Denn die Krise des Wohlfahrtsstaates ist nicht nur finanzieller Art und wird sich nicht einfach auflösen, sobald (oder falls) eine neue Wachstumsperiode beginnt. Die Tatsache, daß die Finanzkrise des Staates weitgehend mit Blick auf die übergroße Last der Sozialausgaben interpretiert wurde, spiegelt eine Verschiebung im Verhältnis von ökonomischen und politischen Kräften wider, die in der allgemeinen Dynamik des Ford ismus wurzelt. Dies bedeutet wiederum, daß die Erneuerung kapitalistischer Expansion keine einfache Rückkehr zum status quo ante bewirken wird. Hinzu kommt, daß die wirtschaftliche Krise des Wohlfahrtsstaates ebenso in der zunehmenden Diskrepanz zwischen seinen Aktivitäten und den Erfordernissen der Kapitalakkumulation begründet liegt. Aufgaben, die während des fordistischen Aufschwungs für die Kapitalakkumulation vorteilhaft waren, haben ein eigenes institutionelles Schwergewicht entwickelt, obwohl sich die Bedürfnisse des Kapitals änderten. Deshalb erfordert die Lösung der ökonomischen Krise nicht nur einen Abbau, sondern auch einen Umbau des Wohlfahrtsstaates.

Zusätzlich zur anhaltenden Krise des Fordismus ist der Wohlfahrtsstaat auch vom Übergang zum Postfordismus betroffen. Denn die Probleme, die bei der Finanzierung des Wohlfahrtsstaates auftreten, werden sich ändern, wenn sich eine neue lange Welle der Akkumulation entwickeln sollte. So wird, selbst wenn etwas ähnliches wie "Vollbeschäftigung« wieder erreicht werden sollte, die Bedeutung von Teilzeit-, zeitlich befristeten und unregelmäßigen Arbeitsverhältnissen viel größer sein als in der fordistischen Periode. Das bedeutet wiederum, daß neue Verfahren der Besteuerung und des Erwerbs von Ansprüchen auf Sozialleistungen eingeführt werden müssen (vgl. Gretschmann 1986; Standing 1986). Gleichfalls wird, mit der wachsenden internationalen Mobilität des Kapitals (besonders im Dienstleistungsbereich) und der wachsenden Konkurrenz zwischen den Staaten um die Ansiedlung von Kapital in Wachstumsbranchen, das Aufkommen aus Kapitalsteuern wahrscheinlich sinken, 
wenn nicht eine koordinierte internationale Politik ent wickelt werden kann. Diese Verschiebungen werden sich auch im politischen Kräfteverhältnis und in der Art von Anforderungen an den Wohlfahrtsstaat widerspiegeln. Allgemein gesagt wird die Notwendigkeit der Flexibilisierung starke Folgewirkungen auf Funktionen und Organisation des Wohlfahrtsstaates haben, wie wir später noch unten sehen werden. Die Krise des Fordismus und der Übergang zum Postfordismus werden so nicht nur das Niveau und die Methoden der Finanzierung des Wohlfahrtsstaates berühren, sondern auch die Formen, in denen der postfordistische Wohlfahrtsstaat seine Funktionen in der gesellschaftlichen Reproduktion erbringen wird.

Unter all diesen Gesichtspunkten ist es irreführend zu behaupten, die fiskalischen, finanziellen und Haushaltsprobleme des Wohlfahrtsstaates könnten getrennt von der Gesamtstruktur der Staatsfinanzen betrachtet werden. Aber die »Neue Rechte« hat es geschafft, diese Problempunkte isoliert darzustellen und die öffentliche Meinung gegen den Sozialstaat zu mobilisieren. Das war mit einer Rücknahme der Verpflichtung zur Vollbeschäftigung verbunden - zuerst mit der Parole der Inflationsbekämpfung und später im Namen der internationalen Konkurrenz. Dies hat den Kompromiß zwischen Kapital und Arbeit untergraben, den beide schlossen, um aus der Vollbeschäftigung in einem Massenproduktions- und Massenkonsumsystem Gewinn zu ziehen.

\section{Die Form des Woblfahrtsstaates}

Wendet man sich dem Wohlfahrtsstaat zu, so ist klar, daß der Wohlfahrtsstaat einige seiner Probleme selbst hervorgebracht hat. Die rational-legale Form der Versorgung mit Sozialleistungen ist verknüpft mit Bürokratisierung, der Verrechtlichung sozialer Beziehungen, politischer Machtballung, Zentralisierung, Klientelismus und der Verstärkung persönlicher Abhängigkeitsverhältnisse. Überdies verschärfen professionalisierte und bürokratisierte Formen von Hilfe und Unterstützung soziale Probleme und verstärken Abhängigkeiten (vgl. bes. Illich). Weiterhin hat die Kombination aus progressiver Besteuerung, Sozialversicherungsansprüchen und bedarfsbezogenen Einzelleistungen zwei Probleme geschaffen: die Armutsfalle, der die Beschäftigten der unteren Lohngruppen gegenüberstehen (Einkommensverbesserungen werden durch Abzüge bei Sozialhilfe etc. konterkariert) und die Arbeitslosigkeitsfalle (die mit der Entwicklung des Realeinkommens zusammenhängt, wenn eine arbeitslose Person eine Arbeit annimmt). Gleichzeitig haben die Formen, in denen Sozialprogramme durchgeführt werden, Verteilungs- und Statuskonflikte innerhalb der Mittelklasse und der Arbeiterklasse verschärft. Oft ist es die Mittelklasse, die soziale Zuwendungen stärker in Anspruch nimmt, speziell die teureren Zuwendungen (z.B. für Bildung, Wohnung, Gesundheit), seien diese vom öffentlichen Wohlfahrtssystem oder durch den sogenannten »fiskalischen Wohlfahrtsstaat «, d.h. auf dem Wege von Steuererleichterungen für gewisse Arten von Konsumausgaben, bereitgestellt. In der Tat dient das Wechselspiel von öffentlicher, fiskalischer und beruflicher Versorgung gleichzeitig dazu, das Ausmaß zu verschleiern, in dem der Staat die soziale Reproduktion der Mittelklasse unterstützt, und neue Brennpunkte für Verteilungs- und Statuskonflikte zu liefern.

Es ist ebenso etwas Wahres an Kritiken, die besagen, der Wohlfahrtsstaat habe eine inhärente Wachstumsdynamik, da soziale Bedürfnisse oft von jenen definiert werden, die ein spezielles Interesse an ihrer Ausdehnung haben. ${ }^{13}$ Das gilt nicht nur für Politiker (angespornt durch den Wahlkampf), Angehörige sozialer Berufe und der Sozialverwaltung (für die eine Ausdehnung des Sozialbereichs neue Arbeitsplätze, Karrierechancen und Ausbau ihres Machtbe- 
reichs bedeutet), sondern auch für Klientelgruppen und politische Lobbies, die ihre Interessen zur Geltung bringen. Dieses Problem wurde akuter, weil die sozialen und ökologischen Kosten der fordistischen Expansion und die Dynamik der Sozialpolitik neue Problembereiche und neue Interessen geschaffen haben, um die herum sich soziale Bewegungen organisieren können.

Zu diesen Problembereichen gehört die sich verschärfende Formkrise der Kleinfamilie, die in der fordistischen Vergesellschaftung sowohl als Ort privaten Konsums wie auch als Hort sozialer und emotionaler Integration in einer atomisierten Gesellschaft eine Schlüsselrolle innehatte (Hirsch und Roth 1986). Der Anteil von Haushalten, die der Kleinfamiliennorm entsprechen, sinkt. Dies spiegelt sich im gestiegenen Bedarf an staatlicher Unterstützung (für Bildung, Krankheit, Alleinerziehende, alte Menschen etc.) wider und schafft die Versuchung, die Lasten für Jugendarbeitslosigkeit, Krankheit und Sorge für die Älteren der Familie aufzubürden. Ebenso signifikant ist der Prozeß des Niedergangs der Innenstädte, der soziale und wirtschaftliche Probleme in Gebieten mit schwindenden Steueraufkommen und wachsendem Bedarf an Sozialausgaben und -programmen konzentriert hat. Insbesondere hier findet man die sozialen Probleme mit Bildung, Wohnen, Gesundheit, Einpersonenhaushalten und Alleinerziehenden, sozialer Isolation und psychischen Krankheiten, sowie demographischen Ungleichgewichten in konzentrierter Form.

In ihrer Folge treten neue Kräfte in der Auseinandersetzung um staat liche Unterstützung auf den Plan. Sie reichen von Kartellen aus Kapital und Arbeit in niedergehenden Industrien und Regionen über ethnische Minderheiten und Alleinerziehende bis zu alternativen kulturellen und sozialen Kollektiven. Die Ausdehnung der »Steuerausgaben « zur Unterstützung der privaten Versorgung mit Gütern und Dienstleistungen (von Renten über Wohnung und Krankenversicherung bis zur Bildung) zur sozialen Reproduktion hat auch neue an sozialpolitischen Leistungen orientierte Interessengruppen unter Steuerzahlern mit angelagerten Kapitalinteressen in diesem Bereich (z.B. Pensionskassen) hervorgebracht. Während der Boomjahre gab es kaum finanzielle oder wahlpolitische Widerstände gegen diese Prozesse, besonders wenn die Jahre des Ausbaus wohlfahrtsstaatlicher Sicherungen mit verringerten Rüstungsausgaben, steigender Produktivität und Vollbeschäftigung einhergingen. Diese Gegengewichte wurden in den letzten Jahren wichtiger. Die Krise des Fordismus ist mit der Finanzkrise des Staates und wachsendem Widerstand der Wähler gegen Besteuerung für Sozialausgaben verknüpft.

Ein weiterer Aspekt ist, daß die monetären und rechtlichen Formen der Sozialpolitik den Problemen, denen sich der Wohlfahrtsstaat heute gegenübersieht, weniger angemessen sind. Ursprünglich hatte er es mit einfachen wirtschaftlichen Wechselfällen zu tun (wie Krankheit, zyklische Arbeitslosigkeit, Schwangerschaft etc.), die das regelmäßige Einkommen von Individuen und/oder Familien unterbrachen. Später expandierte die Versorgung mit grundlegenden sozialen Dienstleistungen, wie Bildung, Wohnen, Gesundheit, und gegenwärtig gewinnen stärker personenbezogene Sozialleistungen und der Umgang mit psychosozialen Problemen (»people-processing «) an Bedeutung. Zusätzlich wurde den tieferliegenden strukturellen Ursachen individueller ökonomischer Wechselfälle (wie den Funktionsbedingungen des Arbeitsmarkts, oder Gesundheit und Sicherheit am Arbeitsplatz) mehr Aufmerksamkeit geschenkt, und die Wirtschafts- und Sozialpolitik wurde auf diesen Gebieten zumindest in den integrierten Wohlfahrtsstaaten besser aufeinander abgestimmt. Eine letzte Veränderung, die es zu erwähnen gilt, ist das Eindringen des Staates in neue Gebiete der Sozialpolitik (wie die Krise der Innenstädte, Rassenbeziehungen und geschlechtsspezifische Benachteiligungen), 
deren komplexere Ursachen im allgemeinen Vergesellschaftungsmuster und nicht einfach in der Wirkungsweise des kapitalistischen Wirtschaftssystems zu suchen sind. Diese Entwicklungen haben das Vertrauen in formale sozialstaatliche Gesetze, bürokratische Organisation und Geldleistungen geschwächt und eine Hinwendung zu gezielter Intervention, professioneller Organisation und zur Versorgung mit Dienstleistungen gefördert. Gerade die jüngeren Trends erschweren es, Sozialpolitik auf rechtliche Ansprüche und/oder formale Methoden versicherungsstatistischer Kalkulation und finanzieller Rationalität aufzubauen (Zacher 1985, S. 26-29). Dies wiederum verlagert die Initiative hin zu Berufs- und/oder Betroffenengruppen, auf Kosten der zentralen und örtlichen Verwaltungen. Dabei werden Erfolgskriterien eingeführt, die nicht nur stärker an Klientelbedürfnissen und/oder professionellen Interessen orientiert, sondern entsprechend auch undurchsichtiger, personalisierter und finanziell ungewisser sind (Vaccarini 1984, S. 124 f.). Außerdem können soziale Probleme wie Rassendiskriminierung oder ungleiche Behandlung der Geschlechter nicht einfach durch eine finanzielle Sicherung gegen soziale Risiken und Mangellagen und/oder durch die Einführung einklagbarer sozialer Ansprüche angemessen gelöst werden. Aber die Alternativen zu formalen, rational-legalen Lösungen bringen ihre eigenen Dilemmata, Widersprüche und Konflikte mit sich. So wird der staatliche Rückgriff auf Sozialtechniken und Sozialprogramme oft durch die Vielfalt und Unbestimmtheit sozialer Ziele sowie durch die Schwierigkeiten, die mit der Antizipation möglicher Auswirkungen solcher Interventionen verbunden sind, verkompliziert. Auch Versuche, die aktive Teilnahme sozial benachteiligter Gruppen oder Gemeinschaften bei der Lösung solcher Probleme zu ermutigen, führen zu wachsenden Ansprüchen, die die Grenzen der Markt - und der bürokratischen Rationalität übersteigen (z.B. Offe 1984).

\section{Sogenannte exogene Faktoren}

Die in der verschränkten Dynamik von Akkumulation und Wohlfahrtsstaat wurzelnden Faktoren wurden durch wichtige exogene Faktoren verstärkt, die zu seiner Krise beigetragen haben. ${ }^{14}$ Darunter stehen demographische Veränderungen an erster Stelle. Diese haben sowohl Umfang wie Finanzen des Wohlfahrtsstaates beeinflußt. Insbesondere hat sich das Verhältnis von Beitragszahlern zu Empfängern in den letzten 20 Jahren dramatisch verändert: wegen des Anstiegs des im Ruhestand befindlichen Bevölkerungsteils (besonders bei den ältesten Kohorten, die einen größeren Bedarf an langfristiger medizinischer Versorgung haben) und eines zahlenmäßigen Rückgangs der Erwerbsbevölkerung. Man sollte ebenfalls erwähnen, daß die Kosten für den Wohlfahrtsstaat tendenziell überproportional stiegen - die Ausbildung dauert länger, der medizinische Fortschritt hat die Kosten gesteigert, Familien von Alleinerziehenden brauchen mehr Unterstützung etc. Das hat den fiskalischen Druck auf die Sozialpolitik verstärkt und die Suche nach Lösungen dringlicher gemacht.

\section{Politische Auswirkungen}

Nichts von alledem bedeutet, daß sich eine unzweideutige und unvermeidliche Logik des Kapitals in irgendeiner Weise durchsetzt. Sogar innerhalb des eigentlichen Arbeitsprozesses ist Raum für signifikante Abweichungen in der "Produktionspolitik «(vgl. Burawoy 1985, sowie Bowles und Edwards 1985). Ebenso können bei der allgemeinen Reorganisation der gesellschaftlichen Struktur der Akkumulation verschiedene Strategien angewandt werden. Das 
kann man nicht nur an den unterschiedlichen Mustern der japanischen, us-amerikanischen, bundesdeutschen und italienischen Wege zum Postfordismus sehen, sondern auch bei den Problemen, die uns hier direkter angehen.

Die Auswirkungen der wirtschaftlichen und politischen Krisen im fordistischen Wohlfahrtsstaat auf das politische Kräfteverhältnis und der Kampf um die Entwicklung konkurrenzfähiger Lösungen sind für die Reorganisation des Wohlfahrtsstaates entscheidend. Darum sollte man den unterschiedlichen Interpretationen der Wohlfahrtsstaatskrise, dem sich verschiebenden Gleichgewicht der politischen Kräfte, die für und gegen seine gegenwärtigen Formen und Funktionen mobilisiert werden, und der Abstimmung zwischen politischen und ökonomischen Strategien besondere Aufmerksamkeit schenken. Die Interpretationen der Krise sind vielfältig. Sie umfassen romantische Zurückweisungen des Wohlfahrtsstaates (erkennbar in den Arbeiten solcher Kritiker wie Illich), Rufe nach einem alternativen, gemeinschaftsorientierten Wohlfahrtsstaat und/oder einem, der sich den Problemen patriarchaler sowie der Klassenherrschaft zuwendet. Hinzu kommen sozialdemokratische Argumente für eine Reorganisation und Einschränkung des keynesianischen Wohlfahrtsstaates für eine begrenzte Periode wirtschaftlicher Austerität, bis dieser, in der mehr oder weniger gleichen Form, wiederhergestellt werden kann, und die Forderungen der "neuen Rechten « nach einer Privatisierung der Sozialleistungen und/oder der Einführung kommerzieller Kriterien in den Wohlfahrtsstaat. Trotz der Bandbreite solcher Kritiken und Lösungsvorschläge ist klar, daß nur eine begrenzte Zahl von Lösungen mit dem Übergang zum Postfordismus vereinbar ist. Wie diese Lösungen kombiniert werden und welche von ihnen sich in den jeweiligen Gesellschaften durchsetzen wird, hängt vom Ausgang politischer und wirtschaftlicher Kämpfe auf dem Terrain unterschiedlicher nationaler Akkumulationsregime und politischer Herrschaftsformen ab. Es ist besser, sich zwei grundsätzliche Alternativen anzusehen, um die herum sich wahrscheinlich Lösungen kristallisieren werden, als fahrlässige allgemeine Voraussagen zu machen.

\section{B. Jenseits der Krise des Woblfahrtsstaates}

Wir können zwei Hauptformen politischer Antworten auf die Krise des Fordismus unterscheiden, die unterschiedliche Konsequenzen für die Politik des Wohlfahrtsstaates haben. Diese Antworten kann man mit dem Etikett "neoliberal« bzw. "neoetatistisch« versehen (vgl. Jacobi u.a. 1985). Am besten sind diese Antworten jedoch als die Pole zu interpretieren, um die sich unterschiedliche nationale Lösungen in einer Periode verstärkter Konflikte und Experimente entwickeln werden, auch wenn es ökonomische, politische und intellektuelle Kräfte gibt, die eng mit der einen oder anderen Antwort identifizierbar sind.

Die neoliberale Antwort beinhaltet eine starke Betonung der Rekommodifizierung (Rückkehr zum Warencharakter) der Arbeitskraft, der Privatisierung von Staatsunternehmen und sozialen Diensten sowie der Deregulierung des privaten Sektors. Aus der Freisetzung von Marktkräften, die diese dreigliedrige Strategie anstrebt, soll demnach die für den Postfordismus entscheidende Flexibilität erwachsen. Insbesondere drängt die neoliberale Antwort auf weitreichende legislative und administrative Veränderungen, um das Kräfteverhältnis auf dem Arbeitsmarkt zugunsten des Kapitals zu verschieben und den Wohlfahrtsstaat in ein Instrument zur Unterstützung und Bezuschußung niedriger Löhne in den Krisenzonen einer Wirtschaft zu verwandeln, die insgesamt durch wesentlich größere Einkommensunterschiede gekennzeichnet sein wird. Empirische Beispiele für diese Politik schließen Versuche ein, 
die Fähigkeit der Gewerkschaften zu effektiven Streikaktionen zu schwächen, korporative Strukturen und Institutionen zu demontieren, die Erwartungen an Lohnniveau und Arbeitsbedingungen zu senken, Flexibilität und Mobilität auf dem Arbeitsmarkt zu fördern und die disziplinierende Wirkung von Maßnahmen und Programmen im Bereich sozialer Sicherung zu verstärken. So soll das Lohnverhältnis wieder als Mittel gesellschaftlicher Reproduktion gestärkt, der Soziallohn gesenkt und den Erfordernissen flexiblerer Beschäftigungsverhältnisse angepaßt werden. Dies beinhaltet sowohl Kürzungen, so daß Einkommenssicherung und andere Sozial programme nurmehr einen minimalen, residualen Stellenwert in der sozialen Reproduktion einnehmen, als auch Reorganisation, so daß Sozialpolitik durch Effizienzkontrolle und differenzierte Zielplanung (z.B. Armut als Folge von Langzeitarbeitslosigkeit, bei Alleinerziehenden oder durch Alter) enger an die wirtschaftliche und soziale Stellung spezifischer Gruppen gebunden ist. Dies erfordert zunehmend mehr Effizienzprüfungen für einen größeren Teil der Sozialausgaben zur Einkommenssicherung und andere Leist ungen. Zugleich werden Steuerkürzungen, die mit einer Senkung der öffentlichen Ausgaben zusammenpassen, mit der Reorganisation des Steuersystems als einem Instrument ökonomischer und gesellschaftlicher Steuerung gekoppelt. So wird das Steuersystem zu einem Instrument, um individuelle Sozialvorsorge über den Markt und/oder mit den Arbeitgebern ausgehandelte berufliche Sozialsysteme zu fördern. Daneben kann es zur Beeinflußung der industriellen Beziehungen (z.B. mittels Steuervergünstigungen für Gewinnbeteiligungsmodelle), des Arbeitsmarkts (z.B. durch Steuervergünstigungen für Unternehmen bei Teilung von Arbeitsplätzen zur Förderung von Teilzeitarbeit) und der individuellen Konsumtion (z.B. durch Steuervergünstigungen für private Krankenversicherungen) benutzt werden.

Überdies wird der neoliberale Wohlfahrtsstaat dort, wo sich gesellschaftliche Sozialleistungen und Programme zur Einkommenssicherung konträr gegenüberstehen, eine »gemischte Sozialwirtschaft « fördern, in der die direkte öffentliche Versorgung abgebaut und private, profitorientierte Dienstleistungen gefördert werden. Zusätzlich wird der Staat gemeinsame Initiativen von Staat und Privatwirtschaft, freiwilligen Gruppen, Wohlfahrtsverbänden, Selbsthilfe usw. unterstützen. Fiskalische, administrative und Haushaltsmaßnahmen sowie direkte Privatisierung werden die Kommerzialisierung der Sozialleistungen und/oder ihre Bereitstellung durch nichtstaatliche Körperschaften fördern. Obwohl ihm eine marginale Rolle als »letzte Zuflucht « bleibt, wird der Staat überdies versuchen, diese Last, wo diese Maßnahmen ineffektiv sind, soweit als möglich auf die Haushalte und »verantwortliche Verwandte« abzuladen. Was das für die »behütende« Rolle der Frauen bedeutet, bedarf keines weiteren Kommentars.

Die soziale Basis dieser Regulationsweise könnte in den Kerngruppen der Beschäftigten der führenden Sektoren der Wirtschaft (High Tech und Dienstleistungen) gefunden werden, zusammen mit jenen Kapital- und Berufsgruppeninteressen, die vom neoliberalen Wohlfahrtsstaat unmittelbar profitieren. Dies hat gleichzeitig zur Folge: a) die Marginalisierung, wirtschaftliche und politische Schwächung einiger Beschäfrigtengruppen im privaten und öffentlichen Sektor, und/oder derjenigen, deren Einkommen großenteils oder ausschließlich von staatlichen Transferzahlungen abhängt, und b) ihre daraus resultierende Unterwerfung unter die neu gestärkten Marktkräfte und/oder die Begrenzung der Staatsausgaben, so daß sie gezwungen werden können, die Kosten des ökonomischen Wandels und der Reorganisation zu tragen. Diese Fragmentierung der arbeitenden und pauperisierten Klassen wird von Bemühungen begleitet sein, eine unterstützende Klasse aus kleinen Geschäftsleuten und anderen Kleinbürgerschichten zu schaffen, und/oder privilegierte Arbeitergruppen in Kleineigentümer und Kapitaleigner zu verwan- 
deln. Das läßt sich an der ideologischen und politischen Bedeutung erkennen, die Kleinbetrieben und verschiedenen Maßnahmen zur Förderung von Hauseigentum, Gewinnbeteiligungs- und Vermögensbildungsmodellen beigemessen wird (Jacobi u.a., 1985; Jessop u.a., 1984; Goldthorpe 1985, S. 336 f.; Davis 1986).

Im Gegensatz dazu würde die neo-etatistische Antwort eine weitere Dekommodifizierung zur Kompensation der Unzulänglichkeiten des Marktes, eine aktive Strukturpolitik zur Verbesserung der Marktkräfte und Regulierungen der Marktkräfte beinhalten. Flexibilität wird durch eine aktive, marktkonforme Strukturpolitik gesichert werden, bei der das Streben nach Flexibilität über ein Interesse an kurzzeitigen Allokationseffekten durch Faktormobilität und flexible Preise (besonders bezogen auf Arbeitsmobilität und Lohnniveau) hinausgeht und eine mittelfristige dynamische Effizienz wichtig wird, die auf einer koordinierten Verbesserung des Ausbildungsstands, der Technologien, der Infrastruktur, des organisatorischen Leistungsvermögens usw. aufbaut. Dies erfordert verstärkte staatliche Aktivitäten in der Ausbildung flexibler Qualifikationen bei den Beschäftigten wie auch in der Flexibilisierung des Arbeitsmarkts. Ein weiteres mögliches Element dieses Modells ist die Einführung eines garantierten Mindesteinkommens, das allen Bürgern unabhängig von Beiträgen oder Beschäftigungsverhältnissen gezahlt wird. In gewisser Weise würde das die Dekommodifizierung der Arbeitskraft weiter stärken und den Trend hin zu universalen sozialen Bürgerrechten, egalitären sozialen Beziehungen und alternativen Haushaltsformen weiter konsolidieren. Aber es könnte auch die Flexibilität des Arbeitsmarkts fördern, ohne die diskriminierenden und marginalisierenden Auswirkungen, die mit dem neoliberalen Modell verbunden sind (siehe dazu insbesondere Standing 1986, vgl. Jordan 1985). Das Steuersystem müßte zur Einführung eines solchen garantierten Mindesteinkommens grundlegend umorganisiert werden, aber die Veränderungen wären sozial weniger spaltend und weniger dazu angetan, die Rolle der Marktkräfte in der sozialen Reproduktion auszudehnen. Wenngleich auch im »neo-etatistischen« Modell eine »gemischte Wohlfahrtsökonomie« gefördert wird, geschieht dies nicht vorsätzlich zugunsten einer expandierenden Rolle des Privatkapitals. Größeres Gewicht wird stattdessen auf Dezentralisierung in Richtung Selbsthilfegruppen, freiwilligen Organisationen, beruflichen Vereinigungen und nichtprofitorientierten Unternehmungen liegen. Die soziale Basis eines neo-etatistischen Modells wird eine weiterentwickelte Form des sozialdemokratischen oder konservativ-korporativistischen Modells sein. Seine zentrale soziale Basis könnte entweder in einer solidarisch verbundenen Bürgerschaft (besonders bei Einführung eines garantierten Mindesteinkommens) mit den organisierten Lohnabhängigen als Kern gefunden werden, oder in einem zersplitterten Ensemble aus verschiedenen ökonomischen, sozialen und politischen Interessen, die vom Übergang zu einer postfordistischen Ökonomie und einer "gemischten Wohlfahrtsökonomie» profitieren, die im neo-etatistischen Sinne modelliert werden könnte. Im letzterem Fall könnte sich ein selektiver Korporativismus entwickeln, in dem Beschäftigte, die mit flexiblen Kenntnissen den Kern des Produktionssystems bilden, repräsentiert und Beschäftigte, die hauptsächlich zeitlich flexibel eingesetzt sind, ausgeschlossen werden. Eine signifikante Verschiebung in der postfordistischen Gesellschaft wird nichtsdestoweniger die gestärkte Rolle von Organisationen und Gruppen sein, die aus den neuen sozialen Bewegungen kommen, welche sich als Antwort auf die gesellschaftliche Krise der fordistischen Akkumulationsstruktur entwickelten. Dies gilt nicht nur für das »neo-etatistische « Modell (was nahelegt, daß der Terminus »etatistisch « in gewisser Hinsicht irreführend ist), sondern auch für die »neue Ehrenamtlichkeit «, der in neoliberalen Wohlfahrtsstaatsentwürfen so sehr das Wort geredet wird. 
In beiden Fällen wird der postfordistische Staat Prioritäten haben, die von denen des fordistischen Staates unterschieden sind. Sowohl in neoliberalen wie in neo-etatistischen Strategien muß der Förderung der Flexibilität Priorität eingeräumt werden. Daher muß die Angebotsseite Vorrang vor der Nachfrageseite haben. Während der fordistische Staat dahin tendierte, sich an die Starrheit der fordistischen Produktion durch ein flexibles Nachfragemanagement anzupassen, muß der postfordistische Staat versuchen, diese Starrheiten mit einer flexibleren Angebotsstrategie und einem rigiden Umgang mit der Nachfrageseite zu überwinden, damit die Sozialausgaben (inklusive Soziallohn) unter Kontrolle bleiben. Das hat signifikante Auswirkungen auf die Zukunft des differenzierten Wohlfahrtsstaates. Als sich die Wirtschaftspolitik des Staates primär auf die Makroebene und die Nachfrage hin orientierte, war es nicht schwer, die soziale Wohlfahrt (besonders die Einkommenssicherung) als separate Politiksphäre zu behandeln. Aber sobald sich die staatliche Wirtschaftspolitik zur Mikroebene und Angebotsseite hin verschiebt, werden die Verknüpfungen von Wirtschafts- und Sozialpolitik entscheidend. Dies wiederum beinhaltet die Notwendigkeit von radikaleren Veränderungen (und keine Politik der kleinen Schritte) im differenzierten (typisch liberalen) Wohlfahrtsstaat, mehr als in den integrierten Wohlfahrtssystemen, die für sozialdemokratische und konservative Regierungen typischer sind. Aber selbst hier werden Veränderungen in der Form des Wohlfahrtsstaates erforderlich sein.

Bei der Betrachtung dieser Veränderungen müssen wir auf die Unterschiede zwischen den erklärten Zielen der Regierungspolitik und der tatsächlichen Arbeitsmarkt- und Sozialpolitik achten. Denn in diesen Arenen herrscht oft mehr Kontinuität als dies Regierungsrhetorik, Gesetzgebungen oder Aktionen nahelegen. ${ }^{15}$ Teilweise rührt dies von den funktionalen Notwendigkeiten gewisser Interventionsformen in der modernen kapitalistischen Wirtschaft her, teilweise ist dies Folge der Institutionalisierung eines politischen Kräftegleichgewichts in den besonderen Formen von Staat und bürgerlicher Gesellschaft. Dennoch gehen Veränderungen vonstatten, und sie beinhalten im Allgemeinen eher eine Neukomposition als die völlige Demontage von Gewerkschaften und Wohlfahrtsstaat. Im Fall des Wohlfahrtsstaates ist es wahrscheinlich, daß das liberale Modell in Richtung des neoliberalen Wohlfahrtsstaatsmodell umgruppiert werden wird, das sozialdemokratische hin zum neo-etatistischen Modell mit einer starken politischen Verpflichtung zu Wirtschaftsdemokratie und einem garantierten Mindesteinkommen. Gesellschaften mit einer konservativen Wohlfahrtsstaatstradition werden wahrscheinlich Elemente beider Modelle verbinden und einen selektiven Korporativismus, rigiden Dualismus oder eine $Z$ wei-Gesellschaften-Struktur aufbauen.

\section{Postfordismus im Woblfahrtsstaat}

Bisher haben wir den Übergang zum Postfordismus in Hinsicht auf seine allgemeinen Auswirkungen auf den Wohlfahrtsstaat untersucht. Nun wollen wir seine Einführung in den Wohlfahrtsstaat untersuchen. Sie hat drei Aspekte: die Flexibilisierung wohlfahrtsstaatlicher Dienstleistungen, das Verhältnis von öffentlichen und privaten Sektoren und die Kosten des Wohlfahrtsstaates. Die Auswirkungen werden auf allen drei Gebieten spürbar sein.

Aglietta hat festgestellt, daß fordistische Techniken auf kollektive soziale Dienstleistungen wie Gesundheitsfürsorge, Bildung und öffentliche Verwaltung nur schwer anzuwenden waren. Eine der Schwierigkeiten, denen sich der Fordismus gegenübersah, war das Element professioneller Arbeit und die Notwendigkeit, Dienstleistung mit sozialer Kontrolle zu kombinieren. Professionelle widersetzen sich sowohl dem Taylorismus als auch dem Fordismus, 
und die disziplinierenden Funktionen des Wohlfahrtsstaates erschweren die Privatisierung. Diese Situation muß mit anderen Dienstleistungsbereichen kontrastiert werden, in die der Fordismus durch die Massenproduktion langlebiger Konsumgüter eindringen konnte, welche dann von den einzelnen Haushalten benutzt und von sekundären Dienstleistungsindustrien gewartet werden. Beispiele dafür liefern die Bereiche Verkehr (das Auto), Unterhaltung (Fernsehen, Video) und Hausarbeit (langlebige Haushaltsgeräte). Dagegen bewirkt ihr langsames Produktivitätswachstum und steigende Reallöhne, daß arbeitsintensive soziale Dienstleistungen die Akkumulation bremsen. Aglietta schloß daraus, daß dieses Problem nur durch »eine radikale Umwandlung der Produktionsbedingungen der Mittel für den kollektiven Konsum « gelöst werden könne, indem die Akkumulation durch eine »massive Umwandlung unproduktiver Arbeit in mehrwertproduzierende Arbeit «angekurbelt wird (Aglietta 1979, S. 157).

Agliettas Analyse liefert einen nützlichen Ausgangspunkt, aber sie muß durch die Untersuchung dreier, miteinander verknüpfter Problembereiche verfeinert werden. Können erstens neue Arbeitsprozesse in Bereiche kollektiver sozialer Dienstleistungen eingeführt werden? Können zweitens neue Produkte in den sozialen Sektor eingeführt werden? Und kann drittens der Übergang zum Postfordismus das Verhältnis von öffentlicher und privater Versorgung mit Sozialleistungen verschieben? Jeder dieser drei Punkte hat einen materiellen und einen disziplinierenden Aspekt.

Auf der Produktionsebene sind es die Informationstechnologien, die entscheidende Möglichkeiten zur Hebung der Produktivität im sozialen Sektor und zu seiner Flexibilisierung anbieten. Dies ist in den Sektoren am klarsten, die mit der Einkommenssicherung durch Geldleistungen befaßt sind - ein Gegensatz zur Bereitstellung von Dienstleistungen im Wortsinn. Aber die Verbindung verschiedener Informationssysteme bietet auch die Grundlage für Privatisierungsmaßnahmen (z.B. bei Dienstleistungen der Arbeitsvermittlung). Auf Produktebene ist die profitable Entwicklung mehrerer neuer Technologien und Industrien (wie z.B. Biotechnologien, pharmazeutische Produktion und Informationstechnologien) von der weiteren Expansion eines reorganisierten Wohlfahrtsstaates stark abhängig.

Überdies könnten neue Produkte die Mischung aus entlohnter Dienstleistung, Maschinerie und unbezahlter Arbeit (wie sie im ehrenamtlichen Sektor, in Selbsthilfegruppen, der Familie im weiteren Sinn, im Haushalt oder von Sozialhilfeempfängern selbst geleistet werden) radikal verändern, analog zu den Veränderungen, die im privaten Dienstleistungsbereich bereits stattgefunden haben. Schließlich sind, indem Informationstechnologien und neue Produkte die Flexibilisierung und Dezentralisierung vorantreiben, Möglichkeiten für den privaten Sektor gegeben, mit dem öffentlichen Sektor zu konkurrieren. An dieser Stelle können wir nur einige kurze Illustrationen zur Stützung dieser allgemeinen Argumente liefern.

Gershuny und Miles (1983) haben verschiedene Gebiete ausgemacht, auf denen postfordistische Techniken den Dienstleistungssektor radikal verändern könnten. Im Gesundheitsbereich zum Beispiel werden Prozeßinnovationen bei existierenden Dienstleistungen folgendes umfassen: Computerisierung der Aufzeichnungen und Büroautomatisierung; effektivere Versorgung mit Lebensmitteln (catering); elektronische Diagnose- und Überwachungseinrichtungen; computergestützte Diagnosesysteme, die »Experten «-Programme nutzen; Fernüberwachung unter Nutzung bestehender Telekommunikationskanäle und die Anwendung von Laserchirugie und ähnlichen Techniken, die kostengünstiger sind. Organisatorische Innovationen werden neue Medikamente und biologische Diagnosemethoden für verschiedene Krankheitsbilder umfassen; neue präventive und einfache diagnostische Dienstleistungen, 
die auf Informationstechnologien aufbauen, Dienstleistungen des medizinischen Umfelds und Diagramme in Gesundheitsdiensten. Gleichzeitig ist ein anwachsender Trend zur Anstellung professioneller Manager anstelle von Angehörigen medizinischer Berufe für die Leitung von Gesundheitseinrichtungen zu beobachten (Blackburn u.a. 1985, S. 183). Im Bildungsbereich wird die Prozeßinnovation über Fernlehrsysteme vonstattengehen, die auf Telekommunikation, Video und Computerausrüstung bauen, und über die Anwendung von Computern bei der sekundären und tertiären Bildung. Auf organisatorischer Ebene wird es neue Bildungspakete für die Erwachsenenbildung und für Lernbehinderte geben; zusätzlich zu den - mit Unterhaltung kombinierten - Bildungspaketen für den häuslichen Gebrauch. Drittens wird es in der allgemeinen Sozialverwaltung Fortschritte bei der Computerisierung von Berichten, Automatisierung von Beratungsdiensten und der stromlinienförmigen Anpassung der Verwaltung geben. Organisatorische Innovationen könnten ebenso die Herausbildung von Selbsthilfegruppen umfassen, die kommunale Dienstleistungen erbringen und vom Staat bessere Dienstleistungen fordern, sowie das Verschwimmen der Grenzen von Polizeiaufgaben, Gemeinwesenarbeit und Überwachungstätigkeit in staatlichen Behörden.

Zweitens entwickeln sich, was die Mischung von unbezahlter Eigenarbeit und bezahlter Arbeit im privaten und öffentlichen Sektors anbetrifft, bereits neue Konsumnormen, die die Kommodifizierung von und/oder Selbstversorgung mit sozialen Dienstleistungen unterstützen, besonders wenn sie um Informationstechnologien herum angesiedelt sind. So wird die präventive Gesundheitsfürsorge (Jogging, Sportzentren, hochwertige Diäten) zunehmend kommodifiziert und gibt zugleich einen Markt für Sportbekleidungs- und Sportartikelindustrien, alternative Medizin und entsprechende Ratgeberliteratur ab. In der Tat »könnten innerhalb der nächsten 20 Jahre expandierende Märkte für den Verkauf von High Tech-Produkten im Gesundheitsbereich entstehen, und zwar nicht nur an oder vermittelt über die formellen Gesundheitsdienste, sondern auch direkt an einzelne Konsumenten im Ausmaß des Massenkonsums« (Blackburn u.a., 1985, S 188).

Kurz gesagt könnten über die verstärkte Kapitalintensität des Wohlfahrtsstaates und den Trend zur Eigenversorgung mit Dienstleistungen die Kosten für soziale Dienstleistungen beschnitten und die Produktivität gesteigert werden. Dies könnte wiederum die Qualität der sozialen Dienstleistungen ohne zusätzliche Kosten verbessern und/oder die Instrumente zur Kostenkontrolle ohne Verringerungen der Dienstleistungen liefern. Das ist da besonders wahrscheinlich, wo ein integrierter Wohlfahrtsstaat mit einer Koalition politischer Kräfte besteht, die noch der Versorgung mit öffentlichen Dienstleistungen verpflichtet ist. Umgekehrt können da, wo eine politische Koalition entstand, die Privatisierung und Sozialabbau bevorzugt, solche Innovationen zur Ausdehnung der Versorgung durch den privaten Sektor, über einen marginalen Wohlfahrtsstaat hinaus, genutzt werden.

Das Ausmaß, in dem neue Techniken und Organisationsmodelle die Flexibilisierung fördern, kann anhand der Entwicklung von »Health Maintenance Organisations (HMOs) in Kalifornien und anderswo in den USA illustriert werden. Es handelt sich dabei um kommerzielle Unternehmen, die Verträge über die Bereitstellung medizinischer Pflege für einen festgelegten Zeitraum zu einem pauschalen Preis abschließen. Sie sind an präventiver Pflege, medizinischer Innovation und der Kontrolle der Kosten interessiert, und sie beteiligen sich, insofern sie um Kunden konkurrieren, an den normalen Formen kapitalistischen Wettbewerbs (für genaue Informationen siehe McRae 1984). Man kann sich die Entwicklung ähnlicher Organisationen auf anderen Gebieten vorstellen, die bisher vom fordistischen Wohl- 
fahrtsstaat beherrscht wurden. Sie könnten entweder als profitorientierte kapitalistische Unternehmen oder als Elemente einer alternativen Wirtschaft geführt werden. Bildungs-, Wohnungs- und Gesundheitswesen sind die drei augenfälligsten Felder solcher Entwicklungen, aber Fälle wie die Privatisierung amerikanischer Gefängnisse legen es nahe, daß viele andere Bereiche staatlicher Intervention entlang ähnlicher Maximen umorganisiert werden könnten. Was diese Beispiele wichtig macht, ist der Eindruck, daß der Wohlfahrtsstaat nicht nur organisatorisch flexibler gemacht werden kann (durch weniger starre bürokratische Formen und mehr Wettbewerb), sondern auch hinsichtlich des Mix von Arbeitskraft, Kapitalausstattung und Eigentätigkeit.

Nichtsdestoweniger zeigt die aktuelle britische Erfahrung, daß eine solche Flexibilisierung aller Wahrscheinlichkeit nach die Notwendigkeit von Restbereichen staatlicher Dienstleistungen nicht beseitigt und nicht insgesamt zu einer Verbesserung der Dienstleistungen führen muß. Die schnelle und profitable Ausdehnung privater Krankenversicherungen war in Großbritannien durch die Tatsache bedroht, daß neue Kunden in Risikogruppen der Arbeiterklasse und bei Älteren gesucht werden mußten. Einige Unternehmen machten Verluste durch die Expansion über die mit niedrigem Risiko behaftete Mittelklasse hinaus. Desgleichen haben die Erfahrungen bei der Privatisierung einfacher Dienstleistungen im Gesundheitswesen, in der Bildung und Kommunalverwaltung gezeigt, daß nicht nur die Kosten gesenkt wurden, sondern auch das Niveau der Dienstleistungen (vgl. Labour Research Bulletin). Diese und andere Beispiele legen nahe, daß das Dilemma zwischen warenförmiger und nichtwarenförmiger Versorgung mit der Einführung des Postfordismus und/oder mit der Privatisierung nicht verschwinden, sondern nur in neuen Formen erscheinen wird.

\section{Zusammenfassung}

Diese Untersuchung hat jüngere Entwicklungen im Wohlfahrtsstaat mit der Krise der kapitalistischen Nachkriegswirtschaft in Beziehung gesetzt. Diese Krise kann nicht nur in rein ökonomischen Begriffen als die Krise eines spezifischen Akkumulationsregimes verstanden werden, sondern muß ebenso im Sinne einer Krise der Regulationsweise und der nationalen Sozialstruktur der Akkumulation erklärt werden. Die allgemeinsten Kennzeichen dieser Krise sind die Erschöpfung des Wachstumspotentials des Fordismus als spezifische Form des Arbeitsprozesses und die Suche konkurrierender Kapitale und Nationalstaaten nach neuen Akkumulationsstrategien. Dies wiederum erfordert das Zurückdrängen des Wohlfahrtsstaates und dessen Reorganisierung, um die Entfaltung eines neuen Akkumulationsregimes zu fördern. In diesem Sinn liefert die Krise des Fordismus den Kontext, in dem die Krise des Wohlfahrtsstaates gelöst werden muß.

Aber das bedeutet nicht, daß die Krise des Wohlfahrtsstaates auf die Krise des Fordismus reduziert werden kann. Stattdessen kann man zwei Phasen in der Entwicklung der Krise des Wohlfahrtsstaates unterscheiden und beide dem allgemeinen Phänomen wiederkehrender Krisen im Wohlfahrtsstaat gegenüberstellen. Denn es gab immer Probleme, was den Umfang und die Verteilung öffentlicher Ausgaben im Wohlfahrtsstaat betrifft, und die spiegelten sich in wiederkehrenden Runden der Ausdehnung und Einschränkung und/oder größerer Betonung der Ausgaben- oder der Kapitalseite des Sozialbudgets wider. Dieses ist überdies letztendlich immer von wirtschaftlichen und politischen $Z$ wängen bestimmt worden. In der Tat ist es alles andere als neu, wenn man unterschiedliche Phasen herausfindet, in denen die Beto- 
nung auf die legitimatorischen oder auf die Akkumulationsfunktionen des Wohlfahrtsstaates gelegt wird. Im Gegensatz dazu könnte man argumentieren, daß die erste wirkliche Nachkriegskrise des Wohlfahrtsstaates aus seiner Eigendynamik entstand und sich durch graduelle Veränderungen seiner Organisationsform, Interventionsmittel und seiner ökonomischen Anbindungen entfaltete. Die erste Reaktion auf diese Krise umfaßte Rufe nach Verrecbtlichung und Rückzug und/oder nach Schritten in Richtung Dezentralisierung und Entbürokratisierung. Die zweite Phase erlebte die Überlagerung dieser ursprünglichen Krise durch die Probleme, die in der Rolle des Wohlfahrtsstaates in der Krise des Fordismus und im Übergang zum Postfordismus wurzelten. Diese zweite Phase hat klare Auswirkungen auf mögliche Lösungen der ersten Krise des Wohlfahrtsstaates. Denn die Art der Reorganisation des Wohlfahrtsstaates kann nicht nur vom Blickwinkel seiner eigenen Dynamik her betrachtet werden, sondern auch vom Blickwinkel seiner Rolle beim Übergang zum Postfordismus. Diese Probleme sind natürlich in allen Fällen durch Unterschiede in den jeweiligen nationalen Ausprägungen des Wohlfahrtsstaatsregimes wie auch der Regulationsweisen überdeterminiert.

Der hier vorgestellte Ansatz erfordert eine gewisse Vorsicht bei der Behandlung von Veränderungen im Wohlfahrtsstaat. Wir erleben gegenwärtig eine Phase des Übergangs, der Experimente und strategischen Interventionen: die endgültige Form des postfordistischen Wohlfahrtsstaates wird erst später sichtbar werden und außerdem von Gesellschaft zu Gesellschaft variieren. Skocpol argumentierte kürzlich folgendermaßen:

"Besonders in einer Periode globaler wirtschaftlicher Schwierigkeiten werden die spezifischen Kennzeichen jedes Nationalstaates und Parteiensystems, und seine aus der Vergangenheit ererbte, besondere Folge von sozialpolitischen Vermächtnissen entscheidend zur Erklärung, ob überhaupt eine 'Krise des Wohlfahrtsstaates' vorliegt, genauer, wie diese Krise definiert ist, und welche Ideen, Bewegungen und Maßnahmen der Führung Alternativen für die Zukunft definieren« (Skocpol 1985, S. 311).

Eine allgemeine Schlußfolgerung ist gerechtfertigt, vielleicht sogar banal. Wenn unsere Argumente zur sozialen Reproduktion und zur Lohnform richtig sind, ist es nicht möglich, den Wohlfahrtsstaat einfach zu demontieren. Wie Claus Offe zum Wohlfahrtsstaat bemerkte, kann »der Kapitalismus weder mit ihm, noch obne ihn existieren. (Offe 1984, S. 153). So lautet denn die entscheidende Frage, wie der Wohlfahrtsstaat umstrukturiert werden wird, und in welchen Grenzen seine Bedeutung reduziert (aus neoliberaler Perspektive) oder ausgedehnt (aus neo-etatistischer Perspektive) werden kann, ohne den Übergang zum Postfordismus ernsthaft zu behindern.

Die quantitativen Grenzen der Kürzungen sind in allen fortgeschrittenen kapitalistischen Gesellschaften, deren Sozialausgaben, auch in relativer Hinsicht, seit dem Aufkommen der ersten Ölkrise nicht drastisch gekürzt wurden (OECD 1984), deutlich sichtbar. Ob dies nur die zeitlich eingegrenzten Kosten für die Durchführung des gegenwärtigen Umbaus widerspiegelt oder impliziert, daß das Sozialbudget immer auf dieser Höhe bleiben wird, ist weniger klar. Die OECD ist sogar optimistisch genug zu behaupten, daß »es möglich sein sollte, die Zugewinne an Absicherung und Zuwendungen, die in den 60ern und 70ern erzielt wurden, beizubehalten und diese in ungefähr dem Wachstumstempo der Wirtschaft zu verbessern, aber nicht schneller« (OECD 1984). Aber diese Behauptung fußt weitgehend auf einer ausschließlichen Befassung mit finanziellen Ressourcen und einer grundlegenden Vernachlässigung der strukturellen Erfordernisse und der sich verändernden ökonomischen und politischen Kräfteverhältnisse, die mit dem Übergang zum Postfordismus verbunden sind. Dieser Übergang umfaßt eine qualitative Verschiebung der wirtschaftlichen und sozialen Prioritäten des Wohlfahrtsstaates und ist 
begleitet vom Zerfall der politischen Koalitionen, die die Wohlfahrtsstaatspolitik der Nachkriegszeit stützten. Die Reorganisierung des Wohlfahrtsstaates beinhaltet Verschiebungen im Umfang der Sicherungen und Leistungen, wie sie in den 60er und 70er Jahren charakteristisch waren, und die Übernahme neuer ökonomischer Funktionen und disziplinierender Aufgaben in der sozialen Reproduktion. Sozialdemokratische Wohlfahrtsstaatsregimes könnten das optimistische Szenario der OECD in den kommenden Jahrzehnten verwirklichen, aber es ist unwahrscheinlich, daß liberale Wohlfahrtsstaatsregime dies tun werden. Letztere werden sich eher in Richtung eines neoliberalen Musters bewegen, das durch ein integriertes Sozialsystem charakterisiert ist, in dem die Angebotsflexibilisierung mit einem Minimum an wohlfahrtsstaatlicher Sicherheit kombiniert ist. Ganz allgemein werden viele der Dilemmata und Widersprüche des fordistischen Wohlfahrtsstaates im postfordistischen System reproduziert werden. So wird die Suche nach alternativen Lösungen für die zentralen Probleme der gesellschaftlichen Reproduktion in einer kapitalistischen Gesellschaft, in der das Lohnarbeitsverhältnis für so viele Produzenten eine Grundgegebenheit des Lebens ist, weitergehen.

\section{Anmerkungen:}

* Die Grundlinien dieses Konzepts haben wir mit dem Beitrag von Alain Lipietz, Akkumulation, Krisen und Auswege aus der Krise: Einige methodische Überlegungen zum Begriff der $»$ Regulation «, in Heft 58/1985 der Prokla vorgestellt (d. Red.).

1 Die Definition einer bestimmten Bevölkerung ist $z$ wangsläufig immer etwas willkürlich und hängt vom jeweiligen Bezugspunkt ab. Man könnte etwa die gesellschaftliche Reproduktion des Gesamtarbeiters, der innerhalb der Grenzen eines Nationalstaates lebenden Bevölkerung, der für militärische Kriegsführung notwendigen Bevölkerung etc. untersuchen.

2 Dabei werden u.a. Phänomene wie Arbeitsmigration, internationale Arbeitsteilung und Imperialismus relevant.

3 Die folgenden Anmerkungen liegen auf der Ebene der rein kapitalistischen Produktionsweise und behandeln die Notwendigkeit staatlicher Intervention für die gesellschaftliche Reproduktion. Sie behandeln weder die Sozialpolitik in Gesellschaften mit unterschiedlichen Produktionsweisen noch die historische oder institutionelle Herausbildung des Wohlfahrtsstaates in einzelnen Gesellschaften.

4 Dies bedeutet jedoch nicht, daß die Arbeiter für ein solches Resultat zu tadeln wären. Konsumnormen entstehen sowohl inner- wie außerhalb des Kapitalkreislaufs.

5 s. Wilensky 1975, S. 1; Donati 1983, S. 57.

6 Vaccarini (1983) gibt einen Überblick über die neueren deutschen und anglo-amerikanischen Ansätze.

7 Diese Skizze bezieht sich vor allem auf: Blackburn et al. 1985; de Vroey 1984; Hirsch und Roth 1986; Hurtienne 1986; Lipietz 1985; Piore und Sabel 1985.

8 fehlt

9 Die Argumentation dieses Abschnitts greift jene von Jacobi u.a. (1985) auf.

10 Das relative Gewicht der beiden Sektoren variiert offenbar je nach nationaler Ökonomie, und dies wird von verschiedenen Mustern von Export- und Importabhängigkeit reflektiert: so dehnt sich der Bereich des Staates bei ihrer Integration auch auf den Wechselkurs und die auswärtige Wirtschaftspolitik aus.

11 Wenn etwas als »soziales Problem « bezeichnet wird, umfaßt das immer politische und ideologische Annahmen zur sozialen Ordnung, der Durchführbarkeit verschiedener Lösungen, dem eigentli- 
chen Umfang staatlichen Handelns usw. Soziale Probleme sind gesellschaftlich hervorgebracht, und das erfordert eine ausgefeilte Analyse der Entstehung von Problemen, für die wohlfahrtsstaatliche Lösungen als angemessen erachtet werden.

12 Dieser Druck wurde so sehr verstärkt, daß der »zweite Kalte Krieg«, der nicht nur ein einfacher Reflex der Krise des Fordismus ist, zu einer Erhöhung der Rüstungsausgaben führte.

13 Aber dieses Argument sollte nicht nur auf den kollektiven Konsum beschränkt werden: Der Fordismus als Ganzes basiert auf der Stimulation neuer Wünsche und Produkte, und privater Konsumismus bedroht durch Lohnforderungen und die Ausweitung von Konsumkrediten die Verwertung genauso stark, wie das sozialer Konsumismus durch Besteuerung und öffentliche Kreditaufnahme tut.

14 Vom Standpunkt der fordistischen Dynamik als Ganzer gesehen, könnte man argumentieren, daß selbst Faktoren wie demographische Veränderungen integrale Bestandteile oder unvermeidliche Folgen einer fordistischen Sozialst ruktur sind. Aber dieses Argument kann für die hier gestellten Anforderungen beiseite gelassen werden.

15 Zu dieser Diskrepanz in den industriellen Beziehungen in der Bundesrepublik und in Großbritannien s. Kastendiek 1985.

\section{Literatur}

Aglietta, Michel (1979): A Theory of Capitalist Regulation, London

Blackburn, Phil/ Coombs, Rod/Green, Kenneth (1985): Technology, Economic Growth, and the Labour Process, London

Bowles, Sam / Edwards, Richard (1985): Capitalism, New York

Boyer, Robert / Coriat, Benjamin (1986): Vortragsmanuskript für eine Konferenz über »Technology, Innovation « in Venedig (März 1986)

Briggs, Asa (1961): The Welfare State in Historical Perspective, in: Archives Européennes de Sociologie, 2 (1961), S. 221-58

Buci-Glucksmann, Christine/Therborn, Göran (1983): Le Defi Social Democrate, Paris

Burawoy, Michael (1985): The Politics of Production, London

Crouch, Colin/Pizzorno, Alessandro (eds.) (1978): The Resurgence of Class Conflict in Western Europe,

2. Bd., London

Davis, Mike (1986): Phoenix im Sturzflug, Berlin

de Vroey, Michel (1984): 'A Regulation Approach Interpretation of the Contemporary Crisis', in: Ca. pital and Class 23

Donati, Pierpaolo (1983): 'Natura, Problemi e limiti del Welfare State: un interpretazione', in: Paolo

Rossi / Pierpaolo Donati (eds.) Welfare State: Problemi e Alternativi, Milano

Esping-Andersen, Gösta (1986): 'Power and Distributional Regimes', in: Politics and Society (1986)

Flora, Peter (1985): 'On the History and Current Problems of the Welfare State', in: S. N. Eisenstadt/

O. Ahimeir (eds.), The Welfare State and its Aftermath, London, S. 11-30

Galbraith, John K. (1967): The New Industrial State, Harmondsworth

Gershuny, Jonathan/Miles, Ian (1983): The New Service Economy, London

Goldthorpe, John (1985): The End of Convergence: Corporatist and Dualist Tendencies in Modern Western Societies, in (ed.): Order and Conflict in Contemporary Capitalism, Oxford

Gretschmann, Klaus (1986): Social Security in Transition: some reflections from a fiscal sociology perspective, Florenz (European University Institute)

Hirsch, Joachim (1985a): Fordismus und Post-Fordismus: die gegenwärtige gesellschaftliche Krise und ihre Folgen, in: Politische Vierteljabresschrift, 26 (Juni)

Hirsch, Joachim (1985 b): Aufdem Weg zum Post.Fordismus?, unveröff. Manuskript

Hirsch, Joachim / Roth, Roland (1986): Das neue Gesicht des Kapitalismus. Vom Fordismus zum Post-Fordismus, Hamburg 
Hurtienne, Thomas (1986): Fordismus, Entwicklungstheorie und Dritte Welt, in: Peripherie, 22/23 (1986)

Jacobi, Otto/Jessop, Bob/Kastendiek, Hans (1985): 'Corporatist and Liberal Responses to the Crisis of Postwar Capitalism', in: idem und Marino Regini (eds.), Capitalist Crisis, Trade Unions, and the State, London

Jessop, Bob (1982): The Capitalist State, Oxford

Jessop, Bob (1983): Accumulation Strategies and Hegemonic Projects, in: Kapitalistate, 10 (1983)

Jessop, Bob (1986): The Mid-Life Crisis of Thatcherism, in: New Socialist, March (1986)

Jordan, Bill (1985): The State: Authority and Autonomy, Oxford

Kastendiek, Hans (1985): 'The Politics of Industrial Relation - a Persistent Diversity of National Patterns", Vortragsmanuskript für "Conference on Labour Exclusion or New Patterns of Cooperation (Institut für Sozialforschung, Frankfurt/M. vom 12.-15.-12. 1985)

Krätke, Michael (1982): Zur politischen Ökonomie des Wohlfahrtsstaates - und ihre überfällige Kritik, in: Prokla 49

Kundig, Bernard (1984): 'Du taylorisme classique a la »flexibilisation « du systeme productif. L'impact macro-économique des differents types d'organisation du travail industriel', in: Critiques del'économie politique, 26/27 (1984)

Lipietz, Alain (1985): Akkumulation, Krisen und Auswege aus der Krise, in: Prokla 58 (1985)

Mazier, Jacques et al. (1985): Quand Les Crises Durent, Paris

Mishra, Ramesh (1984): The Welfare State in Crisis, Brighton

Morville, Pierre (1985): Les nouvelles politiques sociales du patronat, Paris

Müller, Wolfgang/Neusüss, Christel (1970): Die Sozialstaatsillusion und der Widerspruch von Lohnarbeit und Kapital, in: Sozialistische Politik, 2. Jg., Heft 6/7 (1970)

OECD (1984): Social Expenditure: Erosion or Revolution?, The OECD Observer, 126 (1984)

Offe, Claus (1984): Contradictions of the Welfare State, London

Offe, Claus (1985): Disorganized Capitalism, Cambridge

Piore, Michael/ Sabel, Charles (1985): Das Ende der Massenproduktion?, Berlin

Poulantzas, Nicos (1978): Staatstheorie, Frankfurt

Przeworski, Adam (1985): Capitalism and Social Democracy, Cambridge

Skocpol, Theda (1984): What is Happening to Western Welfare States?, in: Contemporary Sociology (1984), S. 307-311

Standing, Guy (1986): Labour Flexibility and Flexibility in Social Security

Tuschling, Burkhard (1976): Rechtsform und Produktionsverbälnisse, Frankfurt

Vaccarini, Italo (1983): La riflessione-della sociologia germanica sul »Welfare State«, in: G. Rossi/P. Donati (eds.), Welfare State: Problemi e Alternavi, Milano

Walker, Alan (1983): Labour's Social Plans: the Limits of Welfare Statism, in: Critical Social Policy, 8 (1983)

Wilensky, Harold L. (1975): The Welfare State and Equality, Berkeley

Zacher (1985): Verrechtlichung im Bereich des Sozialrechts, in: Friedrich Kuebler (Hg.), Verrechtlichung von Wirtschaft, Arbeit und sozialer Solidarität, Frankfurt 